\title{
Genome-Wide Association Study in New York Phytophthora capsici Isolates Reveals Loci Involved in Mating Type and Mefenoxam Sensitivity
}

\author{
Gregory Vogel, ${ }^{1,2}$ Michael A. Gore, ${ }^{1}$ and Christine D. Smart $2, \dagger$ \\ ${ }^{1}$ Plant Breeding and Genetics Section, School of Integrative Plant Science, Cornell University, Ithaca, NY 14853 \\ 2 Plant Pathology and Plant-Microbe Biology Section, School of Integrative Plant Science, Cornell University, Geneva, NY 14456 \\ Accepted for publication 12 June 2020.
}

ABSTRACT

\begin{abstract}
Phytophthora capsici is a soilborne oomycete plant pathogen that causes severe vegetable crop losses in New York (NY) state and worldwide. This pathogen is difficult to manage, in part due to its production of long-lasting sexual spores and its tendency to quickly evolve fungicide resistance. We single nucleotide polymorphism (SNP) genotyped 252 P. capsici isolates, predominantly from NY, in order to conduct a genome-wide association study for mating type and mefenoxam sensitivity. The population structure and extent of chromosomal copy number variation in this collection of isolates were also characterized. Population structure analyses showed isolates largely clustered by the field site where they were collected, with values of $\mathrm{F}_{\mathrm{ST}}$ between pairs of fields ranging from 0.10 to 0.31 . Thirty-three isolates were putative aneuploids, demonstrating evidence for having up to four linkage groups present in more than two copies, and an additional two
\end{abstract}

isolates appeared to be genome-wide triploids. Mating type was mapped to a region on scaffold 4 , consistent with previous findings, and mefenoxam sensitivity was associated with several SNP markers at a novel locus on scaffold 62 . We identified several candidate genes for mefenoxam sensitivity, including a homolog of yeast ribosome synthesis factor Rrp5, but failed to locate near the scaffold 62 locus any subunits of RNA polymerase I, the hypothesized target site of phenylamide fungicides in oomycetes. This work expands our knowledge of the population biology of $P$. capsici and provides a foundation for functional validation of candidate genes associated with epidemiologically important phenotypes.

Keywords: ecology and epidemiology, genetics and resistance, population biology.
Phytophthora capsici is a soilborne oomycete plant pathogen that causes a disease known as Phytophthora blight on several economically important vegetable crops, including pepper, squash, and pumpkin. Phytophthora blight results in serious crop losses via both root and crown rot, which can lead to sudden irreversible wilting of the plant, and fruit rot, which manifests as rapidly expanding sunken lesions featuring a distinctive white sporulation (Granke et al. 2012). Vegetable growers have found $P$. capsici highly difficult to control for several reasons: it produces spores that can remain dormant in the soil for many years (Carlson et al. 2017; Lamour and Hausbeck 2003), rapidly reproduces asexually under suitable environmental conditions (Hausbeck and Lamour 2004), and tends to quickly evolve insensitivity to once effective fungicides (Kousik and Keinath 2008; Parra and Ristaino 2001).

Previous population genetic surveys of $P$. capsici isolates, both in Michigan (Lamour and Hausbeck 2001) and New York (NY) (Dunn et al. 2010), have shown that different agricultural fields feature distinct, sexually recombining pathogen populations with limited gene flow between them. These results are consistent with the biology of P. capsici. Asexual sporangia and zoospores, both shortlived structures that cannot survive desiccation, are limited in their ability to spread quickly, as they are dispersed in water but not wind

†Corresponding author: C. D. Smart; cds14@cornell.edu

Funding: This research was supported by the New York State Department of Agriculture and Markets (grant number C00237GG); USDA National Institute of Food and Agriculture Specialty Crop Research Initiative (2015-51181-24285); and H. M. Clause, who provided support for Summer Research Scholars at Cornell AgriTech.

*The $e$-Xtra logo stands for "electronic extra" and indicates two supplementary tables and supplementary materials are published online.

The author(s) declare no conflict of interest.

(C) 2021 The American Phytopathological Society
(Bowers et al. 1990; Granke et al. 2009; Schlub 1983). Sexual oospores, on the other hand, possess thick walls that allow them to survive harsh environmental conditions. Oospores can overwinter in temperate climates and remain viable in the soil for years (Babadoost and Pavon 2013; Bowers et al. 1990; Carlson et al. 2017; Lamour and Hausbeck 2003).

As a heterothallic species, $P$. capsici only reproduces sexually when isolates of opposite mating type are in contact (Erwin and Ribeiro 1998). The mating types of Phytophthora species, referred to as A1 and A2, do not signify the production of particular sex organs, as isolates of either mating type are hermaphroditic and thus capable of producing both antheridia and oogonia, the male and female reproductive organs, respectively (Ashby 1929; Judelson 1997). Rather, each mating type secretes a specific hormone $\left(\alpha_{1}\right.$ or $\alpha_{2}$ ) that induces sexual reproduction in isolates of the opposite mating type (Ko 1978). Both mating type hormones have been isolated and characterized (Ojika et al. 2011; Qi et al. 2005), and mapping experiments using experimental crosses have identified a single locus controlling mating type in $P$. capsici, $P$. infestans, and P. parasitica (Carlson et al. 2017; Fabritius and Judelson 1997; Lamour et al. 2012). Recently, a genome-wide association study (GWAS) was conducted to identify the mating type locus of another oomycete, the grapevine downy mildew pathogen Plasmopara viticola (Dussert et al. 2020). Researchers working on different oomycete pathogens have hypothesized that mating type inheritance is analogous to sex determination in an XY system, where in the case of $P$. capsici for example, A1 isolates are homozygous (i.e., XX) and A2 isolates heterozygous (i.e., XY) at the mating type locus (Carlson et al. 2017; Dussert et al. 2020; Fabritius and Judelson 1997). Nevertheless, the exact gene or genes conferring mating type remain unknown in all oomycetes.

Once a sexual population of $P$. capsici is established in a field, the eradication of the pathogen is highly unlikely and control strategies must be implemented to manage disease in future years. In addition to cultural practices designed to promote soil drainage and thereby 
deny a suitable environment for $P$. capsici, fungicides are one of the most effective means that growers rely on to control Phytophthora blight (Hausbeck and Lamour 2004). Phenylamide fungicides in particular, first the racemic metalaxyl and later its active enantiomer mefenoxam, have been used extensively since the 1970s due to their systemic activity in plants and high toxicity against many oomycete species via inhibition of ribosomal RNA (rRNA) synthesis (Davidse et al. 1983; Erwin and Ribeiro 1998; Wollgiehn et al. 1984). Shortly after these fungicides were first deployed, however, resistance emerged in populations of several important oomycete plant pathogens (Gisi and Sierotzki 2008). Isolates of $P$. capsici insensitive to mefenoxam were first reported in 1997 in New Jersey, North Carolina, and Michigan (Lamour and Hausbeck 2000; Parra and Ristaino 2001) and have since been found in many additional states, including NY (Dunn et al. 2010; Keinath 2007; Wang et al. 2009). As with mating type, the genes involved in mefenoxam insensitivity in oomycetes are largely unknown.

Once thought by many researchers to be haploid in their vegetative state, as are true fungi, cytological evidence in the 1960s and 1970s established oomycetes as diploid with meiosis occurring in the gametangia immediately prior to fertilization of the oogonium (Sansome 1961; Sansome and Brasier 1973). In recent years, data generated by next-generation sequencing technologies have been increasingly used not only for traditional population genetic analyses, but also for estimating ploidy levels of samples (Farrer et al. 2013; Zhu et al. 2016). As a result, researchers have identified polyploid Phytophthora isolates with three or more copies of each chromosome (Yoshida et al. 2013), as well as aneuploid individuals with a high degree of chromosomal copy number variation within a single genome (Barchenger et al. 2017; Shrestha et al. 2017). Nevertheless, there is still little known about the extent and distribution of chromosomal copy number aberrations within $P$. capsici field populations.

Since 2006, our lab has collected hundreds of isolates of $P$. capsici from vegetable-growing regions of NY State. We set out to leverage this collection in combination with isolates from an additional six states to improve our understanding of patterns of genomic variation in $P$. capsici and to discover loci associated with traits of epidemiological importance. To accomplish this, we genotyped over 200 isolates from our culture collection with genotyping-bysequencing (GBS) (Elshire et al. 2011) and assayed them for their mating type and mefenoxam sensitivity. The specific objectives of this study were to (i) characterize the pathogen population structure in NY and compare findings with expectations based on previous inferences about pathogen dispersal and survival (Dunn et al. 2010), (ii) determine the extent of chromosomal copy number variation among isolates of $P$. capsici collected from the field, and (iii) conduct a GWAS to identify loci involved in the genetic control of mating type and mefenoxam sensitivity.

\section{MATERIALS AND METHODS}

Isolate collection, phenotypic assays, and DNA extraction. Isolates described in this study were either recently collected in NY in 2017 or $2018(n=157)$, obtained from stored cultures isolated from NY sites prior to $2017(n=85)$, or received from out-of-state collaborators $(n=10)$ (Table 1$)$. NY pathogen samples from 2017 and 2018 were isolated from plant parts of several different species with Phytophthora blight symptoms collected at farms and one supermarket (Supplementary Table S1). Small pieces of surfacedisinfected tissue were plated on PARPH medium (Jeffers and Martin 1986) except for samples with evident sporulation, in which case sporangia were directly transferred to the surface of PARPH Petri plates. Plates were then incubated at room temperature for 1 to 2 weeks before transferring a plug from the edge of the growing colonies to new PARPH plates. These plates were sealed with Parafilm (Bemis, Neenah, WI) and stored at room temperature in the dark until used to transfer plugs for isolation of single-zoospore cultures.

NY isolates collected prior to 2017, including nine described in previous publications (Dunn et al. 2010; Parada-Rojas and Quesada-Ocampo 2018) (Supplementary Table S1), were obtained from long-term storage tubes containing sterile water and three to four hemp seeds. Cultures of these isolates were started by plating a

TABLE 1. Number of isolates, unique genotypes, and counts of each mating type and mefenoxam sensitivity class per site ${ }^{\mathrm{a}}$

\begin{tabular}{|c|c|c|c|c|c|c|c|c|c|}
\hline \multirow[b]{2}{*}{ Field } & \multirow[b]{2}{*}{ Region } & \multirow[b]{2}{*}{ Year } & \multirow[b]{2}{*}{ Number of isolates } & \multicolumn{2}{|c|}{ Mating type counts } & \multicolumn{3}{|c|}{ Mefenoxam sensitivity counts } & \multirow[b]{2}{*}{ Number unique genotypes } \\
\hline & & & & A1 & $\mathrm{A} 2$ & $\mathrm{~S}$ & IS & $\mathrm{R}$ & \\
\hline Columbia \#1 & $\mathrm{CD}$ & 2014 & 1 & 0 & 1 & 1 & 0 & 0 & 1 \\
\hline Herkimer \#1 & $\mathrm{CD}$ & 2007 & 1 & 1 & 0 & 0 & 1 & 0 & 1 \\
\hline Rensselaer \#1 & $\mathrm{CD}$ & 2007 & 2 & 1 & 1 & 1 & 0 & 1 & 2 \\
\hline Schenectady \#1 & $\mathrm{CD}$ & 2007 & 2 & 2 & 0 & 1 & 1 & 0 & 2 \\
\hline Schenectady \#2 & $\mathrm{CD}$ & 2007 & 1 & 1 & 0 & 0 & 0 & 1 & 1 \\
\hline Cayuga \#1 & CNY & 2017 & 24 & 9 & 15 & 20 & 3 & 1 & 10 \\
\hline Monroe \#1 & CNY & 2006 & 1 & 1 & 0 & 1 & 0 & 0 & 1 \\
\hline Ontario \#1 & CNY & 2013 & 70 & 34 & 36 & 44 & 1 & 0 & 17 \\
\hline Ontario \#1 & CNY & 2017 & 71 & 34 & 37 & 65 & 4 & 2 & 42 \\
\hline Ontario \#2 & CNY & 2018 & 9 & 0 & 9 & 8 & 1 & 0 & 2 \\
\hline Ontario \#3 & CNY & 2006 & 1 & 0 & 1 & 1 & 0 & 0 & 1 \\
\hline Tioga \#1 & CNY & 2018 & 1 & 0 & 1 & 1 & 0 & 0 & 1 \\
\hline Tompkins \#1 & $\mathrm{CNY}$ & 2018 & 1 & 1 & 0 & 1 & 0 & 0 & 1 \\
\hline Suffolk \#1 & LI & 2018 & 6 & 1 & 5 & 6 & 0 & 0 & 6 \\
\hline Suffolk \#2 & LI & 2018 & 4 & 1 & 3 & 4 & 0 & 0 & 2 \\
\hline Suffolk \#3 & LI & 2018 & 4 & 2 & 2 & 1 & 0 & 3 & 2 \\
\hline Suffolk \#4 & LI & 2018 & 3 & 3 & 0 & 2 & 0 & 1 & 3 \\
\hline Suffolk \#5 & LI & 2007 & 3 & 1 & 2 & 3 & 0 & 0 & 3 \\
\hline Erie \#1 & WNY & 2017 & 17 & 10 & 7 & 9 & 1 & 7 & 8 \\
\hline Erie \#2 & WNY & 2017 & 16 & 10 & 6 & 1 & 6 & 9 & 9 \\
\hline Erie \#3 & WNY & 2018 & 1 & 1 & 0 & 0 & 0 & 1 & 1 \\
\hline Non-NY & NA & NA & 10 & 5 & 5 & 8 & 1 & 1 & 10 \\
\hline NY unknown & NA & NA & 3 & 2 & 1 & 3 & 0 & 0 & 3 \\
\hline Total & NA & NA & 252 & 120 & 132 & 181 & 19 & 27 & 129 \\
\hline
\end{tabular}

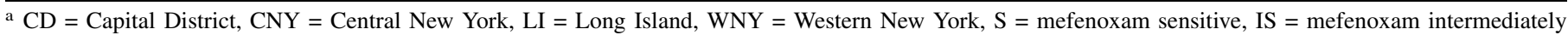
sensitive, $\mathrm{R}=$ mefenoxam resistant. Non-NY isolates were received from California $(n=2)$, Florida $(n=1)$, Michigan $(n=1)$, New Mexico $(n=1)$, Ohio $(n=1)$, and South Carolina $(n=4)$. Mefenoxam sensitivity counts for Ontario \#1 isolates in 2013 are incomplete due to the loss of some cultures in storage prior to performing sensitivity assays. 
sample of the contents of the storage tubes onto PARPH plates. Cultures of non-NY isolates, including one previously described (12889MIA) (Foster and Hausbeck 2009), were received from collaborators and transferred to PARPH plates. All isolates in this study were verified as $P$. capsici by performing PCR with speciesspecific primers (Dunn et al. 2010) and confirming the presence of a band of the correct size via gel electrophoresis.

Single-zoospore isolates were obtained using a protocol similar to that described by Lamour and Hausbeck (2000). Isolates were induced to sporulate by plating on unclarified V8 agar and incubating under $14 \mathrm{~h}$ daily fluorescent lighting for 7 to 14 days. Sporangia were then transferred with a sterile pipette tip to $1.5-\mathrm{ml}$ microcentrifuge tubes containing $1 \mathrm{ml}$ of sterile water and incubated at room temperature for $45 \mathrm{~min}$ to promote zoospore release. Zoospore suspensions were serially diluted and $100-\mu 1$ aliquots of $1: 10,1: 100$, and 1:1,000 dilutions were spread-plated on water agar plates. After incubating at room temperature for approximately 16 $\mathrm{h}$, a stereo microscope was used to identify single germinating zoospores and transfer them to PARPH plates. Single-zoospore cultures were obtained for all but six isolates, which either featured poor colony regrowth (IMK328, FL29, and 568OH) or inadvertently had DNA extracted prior to single zoosporing (1070_3, 14_ 51, and 2014_21).

Mating type and mefenoxam sensitivity assays were performed as described previously (Dunn et al. 2010). Briefly, for mating type determination, isolates were cocultured on unclarified V8 agar plates with NY isolates of known mating type (A1: 0664-1 and A2: 06180-4) (Dunn et al. 2014). After incubating plates in the dark for 7 to 14 days, mating type was ascertained by confirming the presence or absence of oospores in the media using a stereo microscope. For a subset of isolates, consisting of 45 of the 2013 Ontario \#1 isolates and seven isolates from 2006-07 previously characterized by Dunn et al. (2010), mating type was determined at two distinct time points, both prior to entering and after removal from long-term storage. When discordant, the mating type reported and used for analysis was the mating type determined at the time tissue was collected for DNA extraction (corresponding to prior to long-term storage for the 2013 Ontario \#1 isolates and after removal from long-term storage for the 2006-07 isolates).

Mefenoxam sensitivity was determined by transferring $10 \mathrm{~mm}$ plugs from the edge of growing colonies on PARPH to unclarified V8 media amended with mefenoxam at 5 or $100 \mu \mathrm{g} / \mathrm{ml}$ (Ridomil Gold EC; Syngenta AG, Basel, Switzerland). After incubating plates in the dark for 3 days, colony diameters were measured and used to calculate percentage of relative growth (RG) compared with control plates without mefenoxam added. These assays were repeated twice for each isolate and mean $\mathrm{RG}$ values are reported. In order to report a single descriptive summary statistic per isolate, isolates were classified as sensitive $(<40 \% \mathrm{RG}$ on mefenoxam at $5 \mu \mathrm{g} / \mathrm{ml}$ ), intermediately sensitive ( $>40 \% \mathrm{RG}$ on mefenoxam at $5 \mu \mathrm{g} / \mathrm{ml}$ but $<40 \% \mathrm{RG}$ on mefenoxam at $100 \mu \mathrm{g} / \mathrm{ml}$ ), or resistant ( $>40 \% \mathrm{RG}$ on mefenoxam at $100 \mu \mathrm{g} / \mathrm{ml}$ ) as in Silvar et al. (2006).

Mycelia collection and DNA extraction were performed as previously described (Carlson et al. 2017). Plates containing 10\% clarified V8 broth were inoculated with three plugs from the edge of an actively growing culture and incubated in the dark for 4 to 5 days. Approximately $100 \mathrm{mg}$ of mycelia was then vacuum-filtered, collected into 2-ml microcentrifuge tubes containing two zincplated steel BBs (Daisy, Rogers, AR), and stored at $-80^{\circ} \mathrm{C}$ prior to DNA extraction. DNA was extracted using the DNeasy Plant Mini Kit (Qiagen, Valencia, CA) according to the manufacturer's instructions, except a TissueLyser (Qiagen) was used to disrupt mycelia and DNA was eluted into ultrapure water instead of $\mathrm{AE}$ buffer.

Genotyping, single nucleotide polymorphism (SNP) quality control, and clone correction. DNA samples were sent to one of two facilities to prepare and sequence 96-plex GBS libraries digested with ApeKI (Elshire et al. 2011), which had been previously determined to be a suitable restriction enzyme for simultaneously discovering and scoring genome-wide SNPs in P. capsici. Libraries for samples from the Ontario \#1 field site from 2013 were prepared in 2015 at the Institute for Genomic Diversity at Cornell University and sequenced on an Illumina Hiseq2500 generating single-end $100-\mathrm{bp}$ reads. Libraries for remaining samples were prepared in 2019 at the University of WisconsinMadison Biotechnology Center and sequenced on an Illumina NovaSeq6000 generating paired-end 150-bp reads. Replicate samples of isolate 0664-1 were included in order to assess technical variation in genotype calls between GBS plates.

Genotypes were called with the TASSEL GBSv2 pipeline (Glaubitz et al. 2014) using default parameters. To ensure that the same fragments were sequenced for all samples, only the forward reads were used for samples that were paired-end sequenced. Alignment of unique tags to the $P$. capsici reference genome (Lamour et al. 2012) and mitochondrial genome (provided by Martin, F., USDA-ARS) assemblies was performed with the Burrows-Wheeler algorithm bwa-aln (bwa version 0.7.17) with default parameters ( $\mathrm{Li}$ and Durbin 2009). Genotype calls were output in variant call format (VCF) and filtered initially for several criteria using VCFtools version 0.1.17 (Danecek et al. 2011). Indels and SNPs with more than two alleles were removed, as were individuals with greater than $60 \%$ missing data. SNPs that met the following criteria were then retained: (i) mean read depth $>8$ and $<50$; (ii) $>50 \%$ call rate; and (iii) minor allele frequency (MAF) $>0.01$. Genotypes supported by fewer than than reads were set to missing. This SNP set was used for clonal group identification and determination of chromosomal copy numbers.

To identify clonal groups, pairwise identity-by-state (IBS), defined as the proportion of alleles shared at nonmissing sites, was calculated among all isolates. We selected $95 \%$ as a threshold for declaring two isolates as clones, in order to account for an expected genotyping error rate of $\sim 3 \%$ (Carlson et al. 2017), which was consistent with the error rate between technical replicates included among our samples. For all groups of isolates with pairwise IBS that surpassed this threshold, the isolate with the least missing data were retained to create a clone-corrected dataset, which was then further filtered to retain only SNPs with MAF $>0.05$ and call rate $>80 \%$. SNPs that were heterozygous in $>80 \%$ of isolates were also removed, in order to remove likely artifacts of alignment errors. This clone-corrected SNP set was used for all population genetics and GWAS analyses.

All analyses, unless otherwise specified, were performed using custom R scripts (R Core Team 2012; https://github.com/gmv23/ pcap-gwas). Deviations from a 1:1 mating type ratio among the clone-corrected isolate set for each site were tested using an exact binomial test with the $\mathrm{R}$ function binom.test at $\alpha=0.05$. The percentage of variation in RG on mefenoxam-amended media attributed to differences between clonal lineages was calculated from the variance components of a mixed linear model predicting $\mathrm{RG}$, with a random effect for clonal lineage and a fixed intercept term. Models were fit using the R package lme4 (Bates et al. 2015).

Population structure. A principal component analysis (PCA) was conducted using the pcaMethods $\mathrm{R}$ package (Stacklies et al. 2007) on the unit-variance scaled and centered genotype matrix, and using the nipals method to account for missing data. The neighbor-joining tree was created in SplitsTree version 4.14.8 (Huson 1998), using the uncorrected "P" method to estimate the distance matrix, and plotted using the R package ape (Paradis et al. 2004). Values of pairwise $F_{S T}$ between fields, as measured by Weir and Cockerham's estimator of F $_{\mathrm{ST}}$ (Weir and Cockerham 1984), were calculated with the R package StAMPP (Pembleton et al. 2013).

Chromosomal copy number determination. Because there is currently no chromosome-level genome sequence for $P$. capsici, we assigned a total of 97,145 SNPs to the 18 linkage groups of the genetic map provided in Lamour et al. (2012). We then used allele 
balances at heterozygous sites, defined as the number of reads of the major allele divided by the total number of reads, to identify putative linkage groups with more than two copies present in a given isolate, following methods similar to those of Farrer et al. (2013). To minimize variation in allele balances caused by low read depths, we only calculated allele balances at sites supported by 12 reads. For each isolate, SNP allele balances on linkage groups with 30 or more heterozygous SNPs were divided into bins of 47 to $53 \%$ (as expected for a chromosome with two copies) and 30 to $36 \%$ or 63 to $69 \%$ (as expected for a chromosome with three copies). We did not attempt to explicitly identify linkage groups that were present in four or more copies because of the ambiguity in expected allele balances (for example, an allele balance of 0.50 could indicate a copy number of either two or four). Bootstrap analysis was performed 1,000 times over sites and linkage groups were assigned copy number designations if they had over $95 \%$ bootstrap support for featuring predominantly disomic or trisomic allele balances.

Read depth variation across all sites was then used to confirm linkage group copy number designations for putative aneuploid isolates. For each of these isolates, the number of sites on each linkage group was balanced by down sampling to the number of sites on the linkage group featuring the fewest nonmissing SNPs. We then performed an analysis of variance to determine if site read depth varied across linkage groups and Tukey's honest significant difference test to conduct pairwise comparisons across all linkage groups for a given isolate. Putative trisomic linkage groups only retained their trisomic designation if they featured an average read depth significantly different from all putative disomic linkage groups for that isolate at a family-wide confidence level of 0.05 . Fisher's exact test, using the R function fisher.test, was performed to test for significant enrichment for both trisomy across linkage groups and aneuploidy across clonal lineages.

Linkage disequilibrium (LD) calculation and GWAS. Pairwise LD, as measured by $r^{2}$ (Hill and Robertson 1968), was estimated between all SNPs within $500 \mathrm{~kb}$ of each other using the software program PopLDDecay version 3.40 (Zhang et al. 2019). Because the RG phenotype on both mefenoxam-amended media concentrations ( 5 and $100 \mu \mathrm{g} / \mathrm{ml}$ ) had a nonnormal distribution, mefenoxam sensitivity was converted into a binary trait for the purpose of a better fitting model in GWAS (i.e., improved control of Type I error rate). A relatively low threshold, $>10 \% \mathrm{RG}$ on mefenoxam at $5 \mu \mathrm{g} / \mathrm{ml}$, was used to classify an isolate as resistant, in order to separate isolates that were almost entirely unable to grow on mefenoxam-amended media from those that grew to any extent. We did not use the RG on mefenoxam phenotype at $100 \mu \mathrm{g} / \mathrm{ml}$ as it was highly correlated $(r=0.95)$ with RG on mefenoxam at $5 \mu \mathrm{g} / \mathrm{ml}$.

A GWAS for each phenotype was conducted using logistic regression models implemented in the $\mathrm{R}$ package GENESIS (Gogarten et al. 2019). All missing genotype calls were conservatively imputed with the mean value prior to conducting association tests. The Akaike information criterion (Sakamoto et al. 1986) was used to compare the fit of different generalized linear mixed models that included up to four PCs as covariates (to control for population structure) and a random polygenic isolate effect with a covariance structure defined by a kinship matrix (to control for unequal relatedness). The kinship matrix was estimated from the genotypic data using the A.mat function in the R package rrBLUP (Endelman 2011). In the mating type GWAS, we fit a generalized linear model for each SNP that only included a fixed intercept term, as PCs were not selected for inclusion in the model and the random isolate effect (i.e., kinship) was negligible. For mefenoxam sensitivity, SNPs were tested for association in a generalized mixed linear model that included an intercept term, the first three PCs retained as fixed effects, and a random isolate effect. SNPs with a Bonferroni-adjusted $P$ value of $<0.05$ were considered significant. Manhattan and Q-Q plots were created using the R package qqman (Turner 2014).

We used protein-to-protein blast (blastp) to find reciprocal best hits between $P$. capsici and Plasmopara viticola, with the aim of identifying presumable homologs between genes within the $P$. capsici mating type region reported in this study and that reported by Dussert et al. (2020) for Plasmopara viticola. Although Dussert et al. (2019) list a total of 40 genes in the Plasmopara viticola mating type region, we only used the 38 that are annotated in the published Plasmopara viticola reference genome. The complete set of predicted protein sequences of the two oomycete species were aligned against each other using BLASTP+ with default parameters, including a minimum E-value of 10 to save hits (Camacho et al. 2009). A custom python script was then used to identify reciprocal best hits on the basis of E-value.

Data availability. Fastq files containing the raw, demultiplexed GBS reads were deposited in the National Center of Biotechnology Information Sequence Read Archive (BioProject accession number PRJNA616021). Metadata on all isolates, including collection years and locations as well as mating type and mefenoxam sensitivity phenotypes, are provided in Supplementary Table S1. Filtered VCF files for both the nonclone corrected and clone-corrected isolate sets are available at CyVerse (https://datacommons.cyverse.org/browse/ iplant/home/shared/GoreLab/dataFromPubs/Vogel_PcapGWAS_2020). Scripts used for analysis are available on GitHub (https://github.com/ gmv23/pcap-gwas).

\section{RESULTS}

Isolate collection. Between 2007 and 2018, 242 axenic $P$. capsici cultures were isolated from symptomatic plant samples collected at 22 field sites and a supermarket (Tompkins \#1) in NY State (Fig. 1). Geographic areas were represented disproportionately and were sampled in different years, with almost all of the Capital District isolates, for example, collected in 2007. The number of isolates collected from each site also varied considerably, with eleven sites each contributing 1 isolate and another site, Ontario \#1, contributing 141 isolates (Table 1). Ontario \#1 was the only site where samples were collected in two different years, with 70 isolates sampled in 2013 and 71 sampled in 2017.

Isolates were found to belong to both mating types, with a total of 115 A1 and 127 A2 isolates collected (Table 1). A subset of the isolates were assayed for mating type twice, both shortly after collection and after reculturing from long-term storage tubes, up to 13 years later. Of the 52 isolates assayed twice, 13 showed evidence of a mating type switch (Supplementary Table S1). In all 13 of these cases, the mating type was A1 prior to storage and A2 upon reculture.

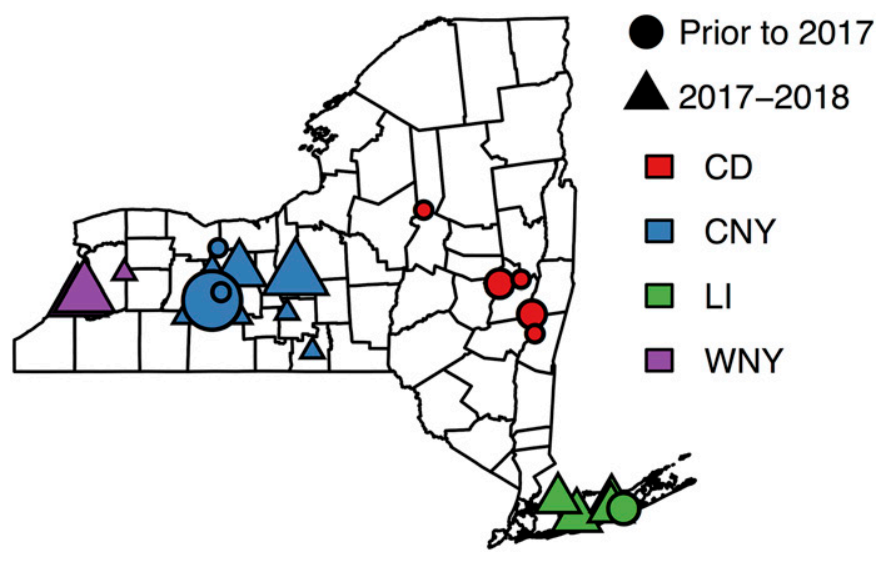

Fig. 1. Map of NY sites where isolates were sampled. Points were randomly assigned a location within the counties where sites were located, with point size indicative of the number of isolates collected per site. One site in Ontario county was sampled twice, in both 2013 and 2017. CD = Capital District, CNY = Central New York, LI = Long Island, and WNY = Western New York. 
Isolates that were intermediately sensitive or resistant to mefenoxam were identified in 12 of the 23 sites across the state (Table 1). Among the seven of these sites where multiple isolates were collected, Erie \#2 featured the greatest proportion of intermediately sensitive or resistant isolates (94\%).

Genotyping and clonality. The $242 \mathrm{NY}$ isolates and 10 isolates from other states (California, Florida, Michigan, New Mexico, Ohio, and South Carolina) were genotyped using GBS. From a total of 363,044 SNPs discovered, initial filtering criteria resulted in a dataset of 107,569 filtered SNPs called on 245 isolates, after removing seven samples with high missing data. This SNP set, featuring a mean SNP read depth with a median of 17.4 calculated across sites and a median of 18.9 calculated across samples, was used to calculate pairwise IBS among isolates in order to identify isolates clonally derived from a common ancestor. Whereas the median IBS between all pairs of samples was 0.741 , pairwise IBS between technical replicates of isolate 0664-1 (including samples sequenced by different facilities) ranged between 0.980 and 0.987 . To account for genotyping error, we therefore used a 95\% IBS threshold for declaring two isolates as clones. The 245 isolates were resolved into 129 unique genotypes (Table 1), 41 of which featured multiple isolates and are hereafter referred to as clonal lineages. A total of 157 isolates belonged to one of these clonal lineages, with a median clonal lineage size of three isolates, and the largest clonal lineage consisting of 17 isolates from Ontario \#1 in 2013. The proportion of isolates that were genetically unique in each site varied, with Ontario \#1 in 2013 (17 unique genotypes out of 70 isolates) and Ontario \#2 in 2018 (two out of nine) featuring the least genotypically diverse samples.

A

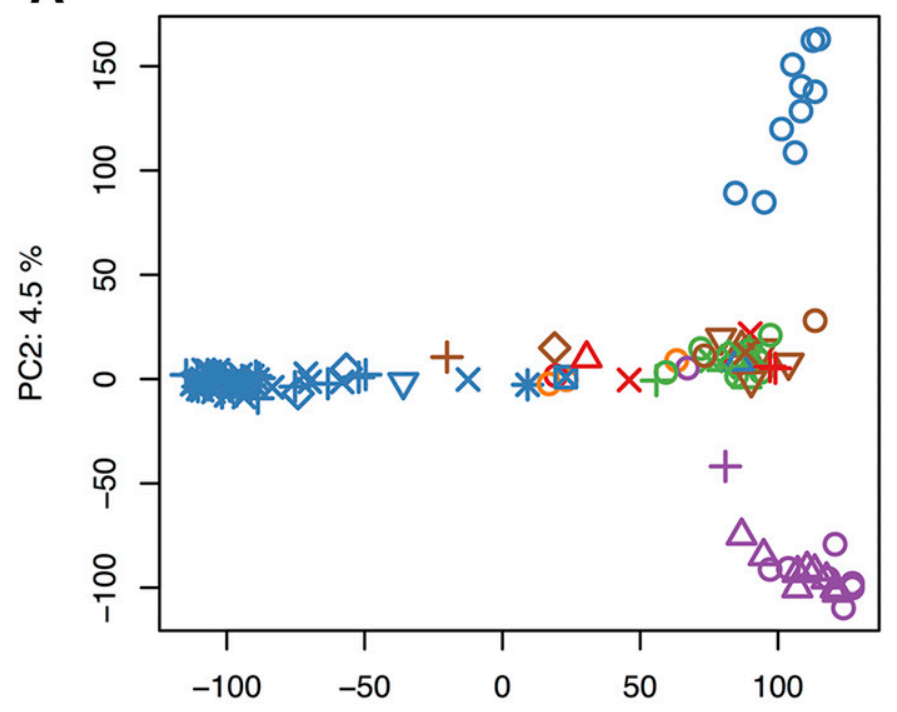

PC1: $15.61 \%$

CD

- Columbia \#1 (2014), $n=1$

$\triangle$ Herkimer \#1 (2007), $n=1$

+ Rensselaer \#1 (2007), $n=2$

$\times$ Schenectady \#1 (2007), $n=2$

$\diamond$ Schenectady \#2 (2007), $n=1$

\section{CNY}

- Cayuga \#1 (2017), $n=10$

$\triangle$ Monroe \#1 (2006), $n=1$

+ Ontario \#1 (2013), $n=17$

$\times$ Ontario \#1 (2017), $n=42$

$\diamond$ Ontario \#2 (2018), $n=2$

$\nabla$ Ontario \#3 (2006), $n=1$

$\otimes$ Tioga \#1 (2018), $n=1$

* Tompkins \#1 (2018), $n=1$

Each clonal lineage was private to a particular site, and in the case of Ontario \#1, none of the clonal lineages identified in 2013 appeared again in 2017. Isolates belonging to the same clonal lineage were largely consistent in terms of mating type and mefenoxam sensitivity. Out of the 41 clonal lineages, only one (consisting of one A1 and two A2 isolates from Ontario \#1 in 2013) featured isolates of opposite mating type. Seven clonal lineages contained isolates with discordant mefenoxam sensitivity classifications, but these only featured a mixture of resistant and intermediately sensitive isolates (four clonal lineages) or intermediately sensitive and sensitive isolates (three clonal lineages). Among all isolates belonging to one of the 41 clonal lineages, 96.1 and $91.7 \%$ of the variation in RG on mefenoxam concentrations at 5 and $100 \mu \mathrm{g} / \mathrm{ml}$, respectively, was attributed to differences between lineages.

A representative isolate was sampled from each clonal lineage to generate a clone-corrected data set consisting of 129 isolates. Using the clone-corrected isolate set, none of the site-years featured a ratio of mating types that differed significantly from 1:1, consistent with the neutral expectation for a sexually reproducing pathogen.

Population structure. In order to visualize genetic relationships among the isolates, we conducted a PCA and created an unrooted neighbor joining $(\mathrm{NJ})$ tree using a high-confidence set of 64,124 SNPs (Fig. 2). PC 1, explaining $15.61 \%$ of the variance in the SNP genotype data, mainly separated the 59 isolates from Ontario \#1 from the remaining isolates (Fig. 2A). PC 2 accounted for $4.5 \%$ of the variance explained and differentiated Western NY isolates from the 10 isolates sampled from Cayuga \#1.

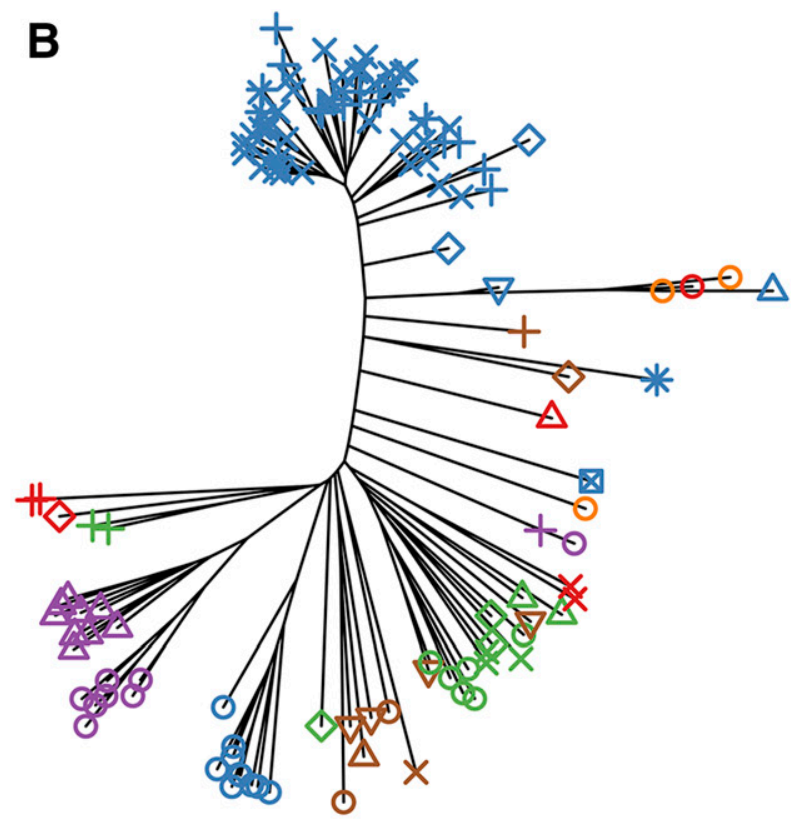

LI

- Suffolk \#1 (2018), $n=6$

$\triangle$ Suffolk \#2 (2018), $n=2$

+ Suffolk \#3 (2018), $n=2$

$\times$ Suffolk \#4 (2018), $n=3$

$\diamond$ Suffolk \#5 (2007), $n=3$

WNY

○ Erie \#1 (2017), $n=8$

$\triangle$ Erie \#2 (2017), $n=9$

+ Erie \#3 (2018), $n=1$
NY unknown

- NY unknown, $n=3$

Non NY

○ CA, $n=2$

$\triangle \mathrm{FL}, n=1$

$+\mathrm{MI}, n=1$

$\times \mathrm{NM}, n=1$

$\diamond \mathrm{OH}, n=1$

$\nabla$ SC, $n=4$

Fig. 2. Population structure of the clone-corrected isolate set. A, Principal component analysis plot showing principal components 1 and 2 calculated from 64,630 single-nucleotide polymorphisms scored on 129 genetically unique isolates. B, Unrooted neighbor-joining tree of the 129 isolates. In both plots, point color reflects geographic region and point shape reflects site within geographic region. 
In the NJ tree, isolates from the same site were largely, but not exclusively, monophyletic (Fig. 2B). Exceptions included isolates from three of the five Long Island sites, as well as the Erie \#1 and Suffolk \#5 populations, each of which featured a single isolate appearing in a distinct clade. The outlier isolate from Erie \#1, collected in 2017, appeared closely related to an isolate sampled from a distinct site in Erie County in 2018 (Erie \#3). Sites from the same region grouped together in several cases, such as with Erie \#1 and Erie \#2 in Western NY and four of the five Long Island sites. However, isolates from different sites in the Capital District did not cluster together on the tree and Tompkins \#1 was clearly separated from the remaining Central NY sites. Non-NY isolates were sorted into several different clades, with even isolates from the same state, such as the four isolates from South Carolina, clustering separately. In both the PCA and the NJ tree, no differentiation was observed between the 2013 and 2017 populations from Ontario \#1.

To quantify differentiation between individual sites, we calculated pairwise $\mathrm{F}_{\mathrm{ST}}$ for the five fields featuring more than five isolates after clone correction (Table 2). Ontario \#1 isolates from 2013 and 2017 were considered jointly as one population due to their genetic similarity $\left(\mathrm{F}_{\mathrm{ST}}=0.001\right)$. Values of $\mathrm{F}_{\mathrm{ST}}$ ranged from a moderate differentiation of 0.10 (Erie \#1 versus Erie \#2) to a very strong differentiation of 0.31 (Cayuga \#1 versus Ontario \#1).

Chromosomal copy number variation. Allele balances at heterozygous SNPs and intragenome variation in read depth were used to infer copy numbers for the 18 linkage groups in each of the 245 isolates with filtered genotype data (Fig. 3; Supplementary Table S2). For a majority of the isolates $(n=222)$, we were unable to assign a copy number to certain linkage groups because they either contained too few heterozygous markers or showed ambiguous signal in their allele balances or read depths (Supplementary Fig. S1). This noisy signal was likely caused, for the most part, by low average read depth, since individual mean read depth and the number of linkage groups assigned a copy number per isolate were highly correlated $(r=0.79)$. Across isolates, the median number of linkage groups assigned a copy number was 13 .

In total, 31 isolates were aneuploid, having between one to four trisomic linkage groups. Linkage groups featuring allele balances suggestive of trisomy mostly featured higher read depths than putative diploid linkage groups, except for four isolates (FL29, 17EH33C, 1070_3, and A2_6_1) where trisomic linkage groups featured lower than average read depth, suggesting a possible tetraploid or higher base ploidy level. Two additional isolates, $17 \mathrm{EH} 20 \mathrm{~A}$ and $17 \mathrm{EH} 21 \mathrm{C}$, were triploid for all of their linkage groups whose copy number we could determine (13 and 16 linkage groups for 17EH20A and 17EH21C, respectively). Although these two isolates were the exclusive representatives of a particular clonal lineage, patterns of aneuploidy were not consistent within clonal lineages for the remainder of the isolates. In fact, among the 10 clonal lineages featuring at least one aneuploid isolate, all trisomic linkage groups were private to individual isolates. There was no significant enrichment for aneuploidy in any of these 10 clonal lineages compared with the rest (Fisher's exact test $P$ value $=0.35$ ).

All linkage groups appeared trisomic in at least one isolate. Considering only linkage groups assigned a copy number designation, Fisher's exact test showed a significant enrichment for trisomies in certain linkage groups compared with others $(P=0.01)$, with linkage groups 6 and 17 displaying the highest rates of trisomy, appearing trisomic in 7 and $8 \%$ of isolates, compared with a linkage group average of $3 \%$ (Table 3 ).

LD decay and GWAS. We used phenotypes and genotypes from the clone-corrected isolate set and conducted GWAS to identify loci associated with mating type and mefenoxam sensitivity. First, we plotted LD between pairwise SNPs as a function of distance, finding that genome-wide LD decayed rapidly to $r^{2}<0.10$ by $\sim 12 \mathrm{~kb}$, and plateaued at $r^{2}=0.04$ by $\sim 400 \mathrm{~kb}$ (Supplementary Fig. S2). We therefore focused on the region within $\pm 400 \mathrm{~kb}$ of peak SNPs identified in GWAS for candidate gene searches.

Mating type showed no relationship with population structure, as it was not associated with any of the first four PCs ( $t$ test $P=0.60$, $0.70,0.90$, and 0.60 for PCs 1 to 4 , respectively) (Fig. 4A). In addition, both mating types were represented approximately equally among the clone-corrected isolate set $\left(\mathrm{n}_{\mathrm{A} 1}=66 ; \mathrm{n}_{\mathrm{A} 2}=\right.$ 63) (Fig. 4B). A logistic regression GWAS for mating type revealed one peak on scaffold 4 containing 70 SNPs with significant $P$ values (Fig. 4C and D). The 70 significant SNPs spanned an approximately $426 \mathrm{~kb}$ region (between SNPs S4_447285 and S4_873767; Supplementary Fig. S3) containing 139 annotated genes, with the peak SNP (S4_579765; $P=4.3 \times 10^{-16}$ ) located in an intron of a gene encoding a putative protein kinase (fgeneshl_pg.PHYCAscaffold_4_\#_142). Among the 70 significant mating type-associated SNPs, A1 isolates were predominantly homozygous, with a median frequency of 0.94 for homozygous genotype calls. A2 isolates, on the other hand, were predominantly heterozygous at these sites, with a median frequency of 0.70 for heterozygous genotype calls.

A total of 290 genes were annotated within $\pm 400 \mathrm{~kb}$ of the peak SNP. Candidate genes were difficult to identify given the unknown molecular mechanism of mating type determination. We used blastp to determine if any of these 290 genes were possible homologs with any of the 38 genes annotated in the recently mapped mating type determining region of Plasmopara viticola (Dussert et al. 2020). Of the 290 P. capsici genes, 199 had a reciprocal best hit in Plasmopara viticola, which were found on 12 different scaffolds, none of them colocalized with the reported GWAS signal for mating type. Likewise, of the 38 Plasmopara viticola genes, 24 had a reciprocal best hit in $P$. capsici, which were found on four separate scaffolds, none of them scaffold 4, where the GWAS signal we identified for $P$. capsici was located.

We next decided to investigate genotype discrepancies between isolates of the clonal lineage which featured one A1 (13EH26A) and two A2 (13EH05A and 13EH76A) isolates. While 13EH26A showed a genome-wide rate of discordant genotype calls between $13 \mathrm{EH} 05 \mathrm{~A}$ and $13 \mathrm{EH} 76 \mathrm{~A}$ of 4.3 and $4.7 \%$, respectively, the discordant call rate was 36.7 and $36.5 \%$ in the $426-\mathrm{kb}$ area spanned by significant mating type-associated SNPs. Considering only the 70 significant SNPs, the discordant call rate was 100 and $97.7 \%$. At 38 of the 42 significant SNPs without any missing genotype calls in these three isolates, 13EH05A and 13EH76A were both heterozygous and 13EH05A was homozygous. In addition to the mating type region on scaffold 4, 13EH26A also showed a higher rate of discordant genotype calls with 13EH05A and 13EH76A on scaffolds 34 and 40 (Supplementary Fig. S4), which immediately proceed the mating type region on the

TABLE 2. Estimates of Weir and Cockerham's $F_{\mathrm{ST}}$ between sites featuring more than five isolates after clone correction ${ }^{\mathrm{a}}$

\begin{tabular}{|c|c|c|c|c|c|c|}
\hline Population & Number isolates & Cayuga \#1 (2017) & Erie \#1 (2017) & Erie \#2 (2017) & Ontario \#1 $(2013,2017)$ & Suffolk \#1 (2018) \\
\hline Cayuga \#1 (2017) & 10 & - & 0.20 & 0.22 & 0.31 & 0.18 \\
\hline Erie \#1 (2017) & 8 & - & - & 0.10 & 0.30 & 0.14 \\
\hline Erie \#2 (2017) & 9 & - & - & - & 0.30 & 0.16 \\
\hline Ontario \#1 $(2013,2017)$ & 59 & - & - & - & - & 0.26 \\
\hline Suffolk \#1 (2018) & 6 & - & - & - & - & - \\
\hline
\end{tabular}

a Ontario \#1 populations from 2013 and 2017 were collapsed into one population. 
P. capsici genetic map (Lamour et. al. 2012). Collectively, these results suggest that a loss of heterozygosity event in a large chromosomal region containing the mating type locus induced a switch from A2 to A1 in isolate 13EH05A. Since the read depth of SNPs in 13EH05A in the mating type region (mean 23.1) did not differ from the genome-wide SNP read depth (mean 22.6), it appears that this loss of heterozygosity event was conferred in a copy-neutral manner.

In contrast to mating type, mefenoxam sensitivity was more strongly confounded with population structure, showing a significant association with PC $1\left(P=5.9 \times 10^{-6}\right)$ and PC $3(P=4.2 \times$ $\left.10^{-3}\right)$. Intermediately sensitive and resistant isolates were especially prevalent in populations from WNY sites, and rare among the 59 isolates from Ontario \#1 (Fig. 5A). In addition, different phenotypic classes were disproportionately represented, with the majority of clone-corrected isolates classified as sensitive (Fig. 5B). We used $10 \% \mathrm{RG}$ on mefenoxam-amended media at $5 \mu \mathrm{g} / \mathrm{ml}$ as a threshold to classify isolates as one of 34 resistant "cases" or 91 sensitive "controls" and conducted a logistic regression GWAS controlling for population structure and unequal relatedness. We identified one signal spanning approximately $59 \mathrm{~kb}$ on scaffold 62 (between markers S62_139120 and S62_198531; Supplementary Fig. S4) that contained six significantly associated SNPs (Fig. 5C and D). The peak SNP $\left(\right.$ S62_186715; $\left.P=1.1 \times 10^{-7}\right)$ was located in an exon of a gene $(g w 1.62 .46 .1)$ annotated as a dynein. The entirety of scaffold 62 was contained within the $400 \mathrm{~kb}$ flanking the peak SNP and featured 100 annotated genes, including several with a plausible role in mefenoxam insensitivity, such as a homolog of the yeast ribosome synthesis factor Rrp5, located within $20 \mathrm{~kb}$ of the peak SNP (Supplementary Table S3).

The allele associated with insensitivity at the peak SNP, S62 186715 , showed an approximately additive effect on RG on both mefenoxam at 5 and $100 \mu \mathrm{g} / \mathrm{ml}$ (Fig. 6). The insensitive allele, with a frequency of 0.28 among all isolates, was found in isolates from 11 NY sites and in one South Carolina isolate. Eight isolates, representing five different field sites in four NY regions, were homozygous for the insensitive allele. Several isolates were outliers in terms of their sensitivity response as predicted by the genotype of SNP S62_186715, including one isolate from Erie \#1 that was homozygous for the sensitive allele yet showed $>95 \% \mathrm{RG}$ on mefenoxam at $5 \mu \mathrm{g} / \mathrm{ml}$ and $>75 \% \mathrm{RG}$ on mefenoxam at $100 \mu \mathrm{g} / \mathrm{ml}$, as well as an isolate from Suffolk \#5 that was homozygous for the insensitive allele yet did not grow on mefenoxam-amended media at either 5 or $100 \mu \mathrm{g} / \mathrm{ml}$.

A
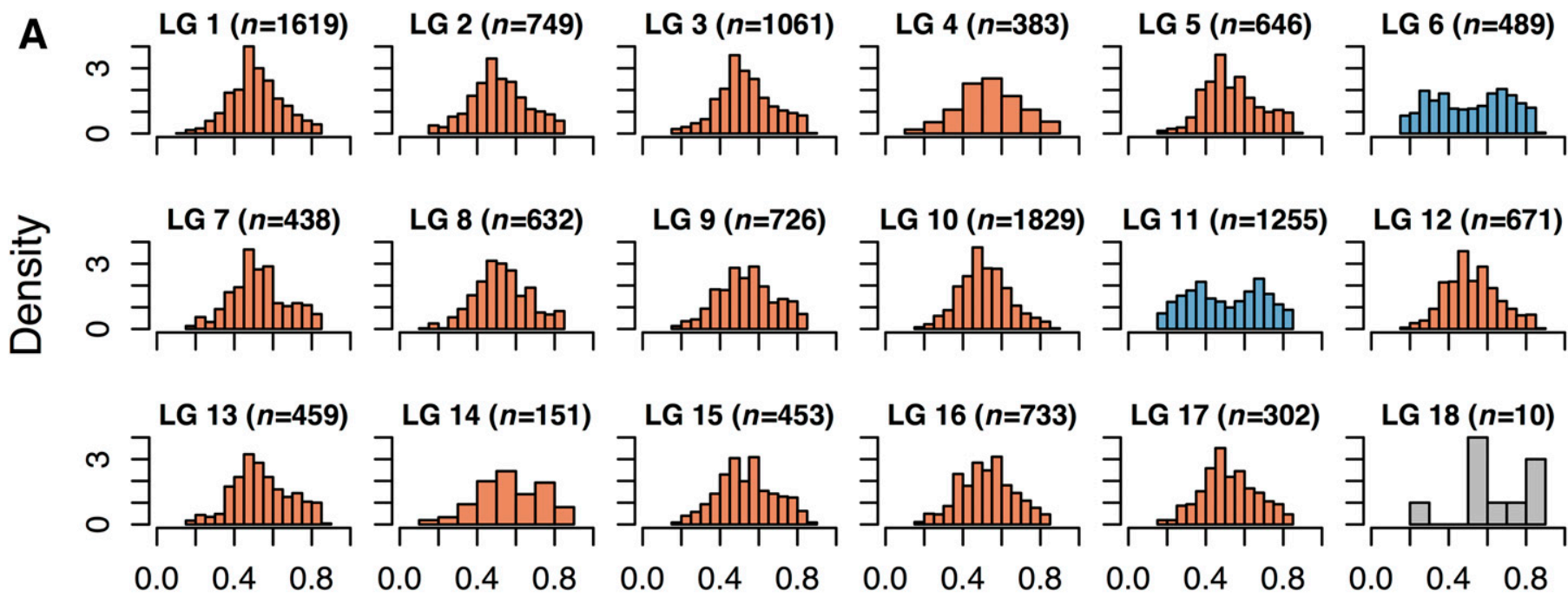

\section{Allele balance}

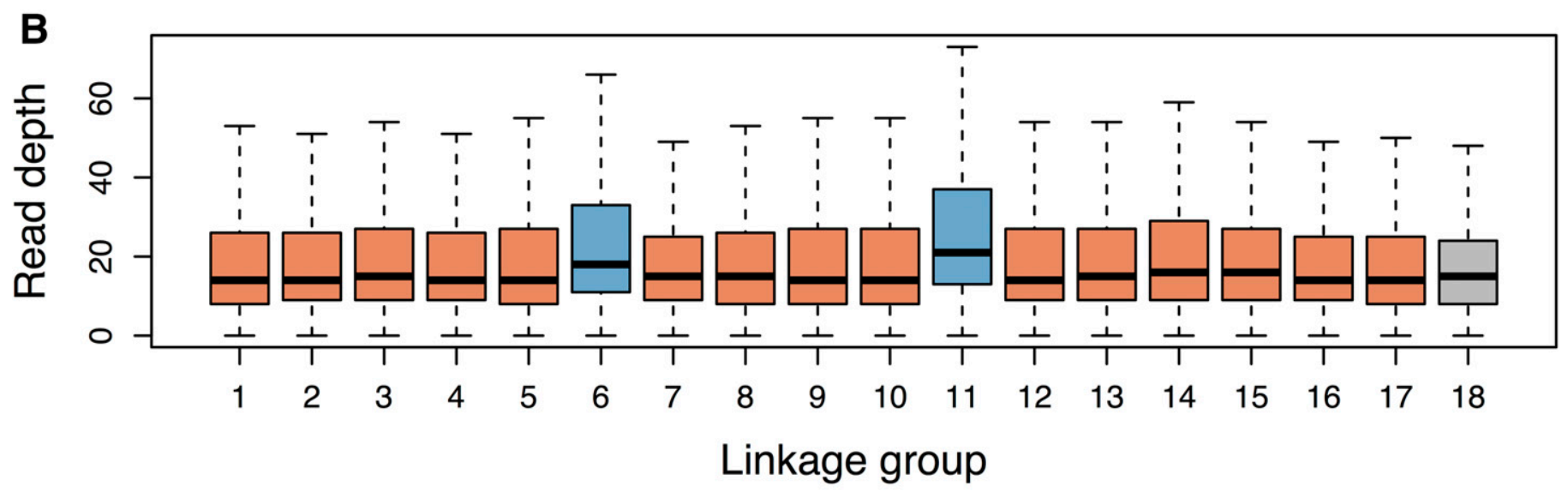

\section{NA

Fig. 3. Example of ploidy level determination by linkage group (LG) in isolate 13EH38A. A, Distribution of allele balances within the 18 linkage groups of isolate 13EH38A. Number of heterozygous markers $(n)$ is reported for each linkage group. B, Boxplot of single nucleotide polymorphism (SNP) read depths per linkage group in isolate 13EH38A. The number of SNPs on each linkage group was down-sampled to the number on the linkage group having the fewest SNPs. 


\section{DISCUSSION}

The 252 P. capsici cultures characterized in this study were isolated from symptomatic plant samples obtained through several means-whether collected during field visits or received from extension agents for purposes of disease diagnostics. We genotyped this collection of isolates at 107,569 SNP loci, corresponding to one SNP on average every $\sim 600 \mathrm{bp}$. We were able to leverage this dataset to investigate not only issues of regional importance, namely those related to the pathogen's population structure patterns in NY, but also questions of global significance, having to do with the genetic control of mating type and mefenoxam sensitivity, both epidemiologically important phenotypes in many oomycete pathogens, and the extent to which field isolates vary in terms of their chromosomal copy numbers.

Population structure and clonality. Visualizing relationships among the clone-corrected isolates with PCA and the NJ tree (Fig. 2) showed, for the most part, pathogen populations from different farms clustering separately. Similarly, estimates of pairwise $F_{S T}$ between the five fields that featured a sufficient number of samples after clone correction showed moderate to strong genetic differentiation between sites. Even isolates from farms in close geographic proximity, such as Erie \#1 and Erie \#2, separated into distinct, although related, clades in the NJ tree and featured moderate levels of genetic differentiation between them. These results agree with previous hypotheses that limited gene flow occurs between $P$. capsici populations on different farms (Dunn et al. 2010; Lamour and Hausbeck 2001).

Nevertheless, we found some examples that deviated from this overall trend. Isolates from three of the five Long Island farms did not separate into distinct clades in the NJ tree, suggesting higher gene flow between the sampled fields in this region. We also identified two isolates, one each from Erie \#1 and Suffolk \#5, that did not cluster with other isolates from their respective sites. It is possible that these outlier isolates represent independent introductions of inoculum to their fields compared with the rest of the isolates from those sites.

As expected based on our current understanding of $P$. capsici epidemiology (Dunn et al. 2010; Lamour and Hausbeck 2001, 2003), we found evidence for a mixed mode of reproduction in pathogen populations in NY fields. Over half of the isolates that we surveyed belonged to a clonal lineage consisting of multiple sampled isolates, reflecting the influence of asexual reproduction within single seasons and fields. Two fields, Ontario \#1 (2013) and Ontario \#2 (2018), featured a particularly small number of genetically unique isolates compared with the total number of isolates sampled from those fields (Table 1). Interestingly, within a few months prior to sample collection, both of these fields experienced flooding events followed by their first outbreak of Phytophthora blight. One possible explanation for their low observed genotypic diversity is that the inoculum, likely introduced to these fields via floodwater, consisted of a small number of founding pathogen genotypes. Alternatively, the environmental conditions in the fields after flooding, highly conducive to asexual reproduction, could have amplified the ability of certain clonal lineages to rapidly spread and predominate in these fields, either due to random chance or because of a fitness advantage of those genotypes.

Isolates from Ontario \#1 were sampled again in 2017, at the end of a season in which the field was planted for the first time since 2013 to a Phytophthora blight-susceptible crop and subsequently suffered another disease outbreak. The 2017 isolates, which were genetically undifferentiated from the 2013 isolates $\left(\mathrm{F}_{\mathrm{ST}}=0.001\right)$ (Fig. 2), were more genotypically diverse, with $59 \%$ of isolates representing unique genotypes compared with $24 \%$ in 2013 . Given that the primary inoculum for the 2017 outbreak was most likely oospores formed during the 2013 outbreak, these results show how a potentially small inoculum founder event followed by sexual reproduction can lead to the development of a persistent bank of genotypically diverse oospores in the soil. This phenomenon has been previously demonstrated in both natural populations (Lamour and Hausbeck 2003) and experimentally inoculated research fields (Carlson et al. 2017; Dunn et al. 2014).

It is unknown when $P$. capsici inoculum first arrived in vegetablegrowing areas of NY, nor where it originated from, or how many times it was independently introduced. Our population structure analyses did not show a clear relationship between NY isolates and those from any particular other state (Fig. 2), although our non-NY sample size $(n=10)$ was too modest to directly address this question. However, other studies have also shown only moderate differentiation between $P$. capsici populations from most U.S. states (Parada-Rojas and Quesada-Ocampo 2018; Quesada Ocampo et al. 2011), suggesting perhaps a recent common origin of inoculum for most states or frequent movement of inoculum between distant locations in the United States. Our data, interpreted in conjunction with other studies (Dunn et al. 2010; Jones et al. 2014; Lamour and Hausbeck 2000, 2001, 2003) and reports from farmers, are consistent with a model of disease spread where local dispersal of inoculum, perhaps largely via run-off from fields and flooding events, leads to long-lasting sexual populations on newly infected fields that become genetically differentiated from neighboring populations due to a founder effect and subsequent genetic drift. Longer-distance movement of inoculum, mediated by transport of infested soil or infected plant material, may also occur, as evidenced by our observation of several field populations with little relatedness to other populations in the same region (e.g., Cayuga \#1).

Two observations from our collection of isolates-that A1 and A2 isolates were represented proportionally in all sites after clone correction, and that clonal lineages were entirely unique to individual sites and years-reflect the epidemiological importance of sexual reproduction in allowing inoculum to overwinter. While obligate sexual reproduction appears to be the case in NY and other U.S. regions (Lamour and Hausbeck 2000, 2003), locations in South America and East Asia, including regions of China with temperate climates that should prohibit overwintering of asexual inoculum, have reported asexual lineages of $P$. capsici that persist over many years and over large geographic regions (Hu et al. 2013; HurtadoGonzalez et al. 2008). It is unknown what environmental and/or

TABLE 3. Distribution of copy number counts for each linkage group (LG) across 245 isolates $^{\mathrm{a}}$

\begin{tabular}{lrrrrc}
\hline Linkage group & $2 n$ & $3 n$ & Unknown & NA & Proportion $3 n$ \\
\hline 1 & 214 & 2 & 27 & 2 & 0.01 \\
2 & 174 & 3 & 65 & 3 & 0.02 \\
3 & 179 & 3 & 60 & 3 & 0.02 \\
4 & 156 & 3 & 82 & 4 & 0.02 \\
5 & 185 & 3 & 54 & 3 & 0.02 \\
6 & 138 & 11 & 91 & 5 & 0.07 \\
7 & 101 & 2 & 138 & 4 & 0.02 \\
8 & 191 & 4 & 47 & 3 & 0.02 \\
9 & 168 & 2 & 72 & 3 & 0.01 \\
10 & 208 & 3 & 31 & 3 & 0.01 \\
11 & 186 & 4 & 52 & 3 & 0.02 \\
12 & 161 & 4 & 77 & 3 & 0.02 \\
13 & 152 & 3 & 87 & 3 & 0.02 \\
14 & 130 & 2 & 108 & 5 & 0.02 \\
15 & 130 & 4 & 107 & 4 & 0.03 \\
16 & 178 & 4 & 60 & 3 & 0.02 \\
17 & 131 & 11 & 99 & 4 & 0.08 \\
18 & 57 & 3 & 110 & 75 & 0.05 \\
Sum & 2,839 & 71 & 1,367 & 133 & NA
\end{tabular}

a Reported are the number of isolates in which a particular LG showed strong statistical support for the presence of two copies $(2 n)$ or three copies $(3 n)$, as well as the number of isolates where that LG showed ambiguous signal (Unknown) or featured too few heterozygous markers for analysis (NA). The proportion of trisomic isolates per linkage group is also reported among isolates where that LG was assigned a copy number (Proportion $3 n$ ). 
genetic factors account for the stark differences in the pathogen population structure between the United States and these other countries.

Chromosomal copy number variation. By analyzing patterns of variation in read counts across the genome, we identified some isolates with clear evidence of aneuploidy (Fig. 3), as well as others with much more difficult-to-interpret signal (Supplementary Fig. S1). We therefore decided to implement conservative thresholds for declaring a linkage group trisomic, relying on significance testing (whether parametric or bootstrap-based) with two independent sources of information, allele balances at heterozygous sites and total read depth at all SNPs. Low average read depth, causing noisy allele balance histograms, was likely responsible for many of the linkage groups with ambiguous support for an allele balance mode at either 0.5 or $0.33 / 0.66$, as evidenced by the high correlation between individual read depth and the percentage of linkage groups assigned a copy number assignations in each isolate. However, it is also possible that linkage groups in some isolates showed noisy allele balance histograms for one of several biological reasons, whether due to possessing an even higher copy number than three, having intrachromosomal copy number variation, or because DNA was extracted from a heterogenous population of nuclei varying in copy number for that linkage group. We therefore may have underestimated the true extent of aneuploidy in our collection of isolates, and future efforts with higher sequencing depth are necessary to expand upon our research. It is also worth mentioning that some of the linkage groups we declared trisomic may actually have been present in greater than three copies, as higher copy number linkage groups could feature allele balance patterns consistent with our definition of trisomy (for example, the tail of an allele balance peak at 0.75 , as would be expected for a triplex tetraploid genotype, could cause a greater number of SNPs to have an allele balance closer to 0.66 than 0.50 ). Finally, our analyses do not rule out heterokaryosis (the presence of multiple distinct nuclei within a single cell), which was recently shown in the oomycete plant pathogen Bremia lactucae to result in similar allele balance distributions to those that we found (Fletcher et al. 2019). However, whereas B. lactucae produces multinucleate sporangia that germinate directly, we have observed $P$. capsici sporangia to almost exclusively germinate indirectly from mononucleate zoospores (data not shown), making heterokaryosis unlikely.

We found 33 isolates, representing $13 \%$ of the isolates we genotyped, that were either aneuploids or genome-wide polyploids. Other studies have reported both widespread polyploidy (Daggett et al. 1995) and aneuploidy (Barchenger et al. 2017; Shrestha et al. 2017) in isolates of P. capsici and other Phytophthora species. However, the number of isolates we genotyped and our detailed analyses allowed us to make several novel findings. First, we found a slight, but significant, enrichment for trisomy in certain linkage groups compared with others. It is unclear if this is due to chromosome size, since the physical size of the $P$. capsici chromosomes is unknown, or has to do with the presence of adaptive genes on certain linkage groups that confer a fitness advantage when present in higher copy number. Second, analyzing patterns of aneuploidy and polyploidy within clonal lineages
A

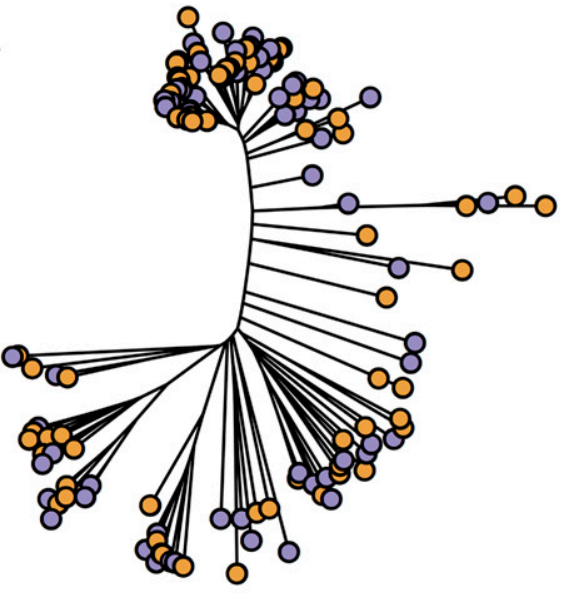

B

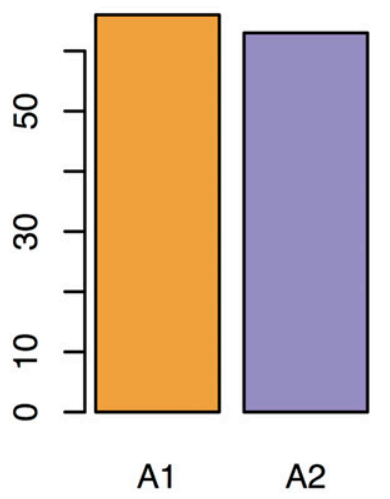

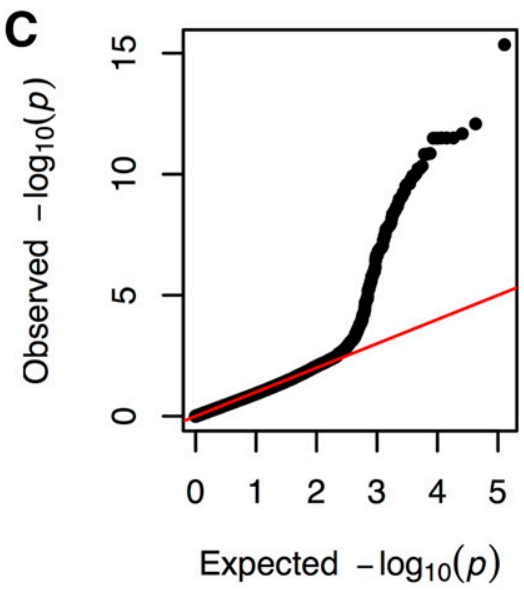

D

ำ

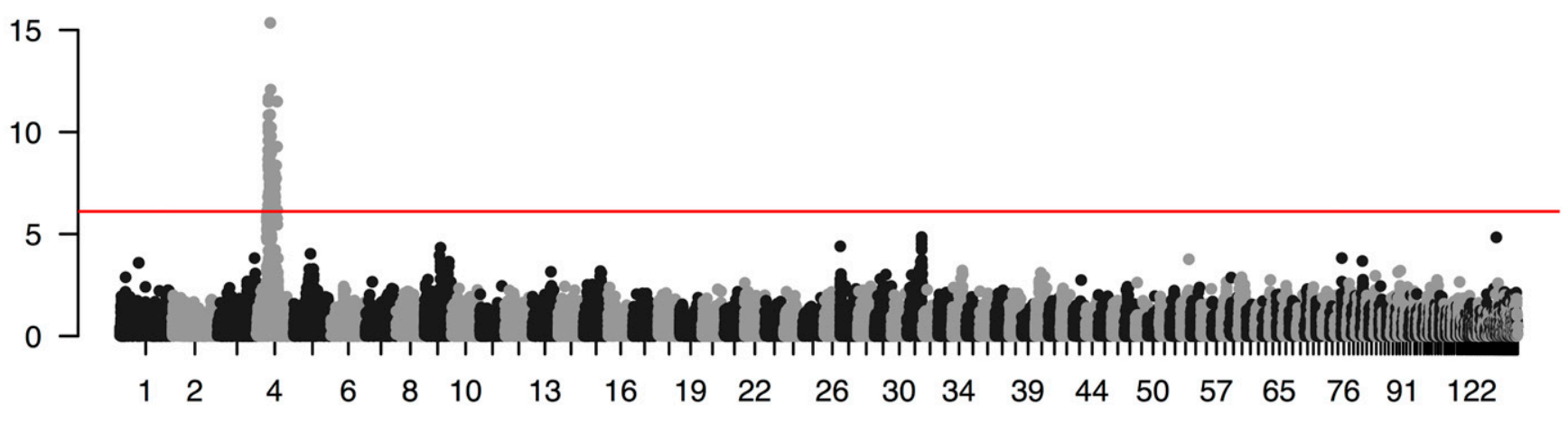

Scaffold

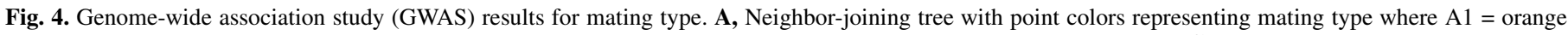

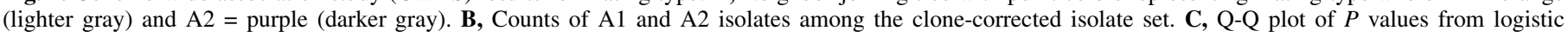

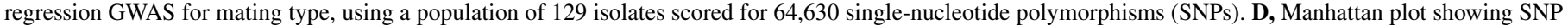
$P$ values across the genome from logistic regression GWAS for mating type. 
showed evidence for a meiotic origin of polyploidy, as the only two genome-wide polyploids we found were the exclusive members of the same clonal lineage, and a mitotic origin of aneuploidy, since trisomic linkage groups were not shared in any cases between isolates of the same clonal lineage. Spontaneous chromosomal loss and duplication in Phytophthora species has previously been identified during vegetative growth and asexual reproduction (Hu et al. 2020; Kasuga et al. 2016), supporting aneuploidy arising mitotically. In our study, differences in aneuploidy within isolates of the same clonal lineage, which presumably originated from a single oospore germinating the same year as sample collection, suggest that chromosomal copy number variation arises rapidly. However, we cannot separate chromosomal duplications that occurred in the field from those that occurred in culture. Future efforts sequencing pathogen DNA directly from field samples would be necessary to understand the rate at which aneuploidy arises and under what conditions.

Aneuploidy is known to be either lethal or causative of severe development defects in many higher organisms (Siegel and Amon 2012). Nevertheless, under selective conditions, experimental evolution studies have shown that aneuploid yeast strains often have higher fitness than euploids (Sunshine et al. 2015). Indeed, in the sudden oak death pathogen $P$. ramorum, aneuploid isolates with phenotypic alterations are consistently selected for after passage through certain hosts (Kasuga et al. 2016). Because of the small number of isolates in our collection having aneuploidy for any given linkage group, we were not able to robustly associate chromosomal copy number changes with either of the traits we phenotyped. However, we hypothesize that spontaneous chromosomal loss and duplication leads to phenotypic variation, even within clonal lineages of $P$. capsici, that are subject to selection. It is unclear, however, if selection for certain aneuploidies persist beyond a single year, given that $P$. capsici undergoes obligate sexual reproduction in order to overwinter in the United States and the ability of aneuploid isolates to undergo meiosis and form viable gametes is unknown.

Genetic basis of mating type and mefenoxam sensitivity. To our knowledge, our study represents the first GWAS conducted in $P$. capsici, and one of few ever conducted in a plant-pathogenic oomycete (Ayala-Usma et al. 2020; Dussert et al. 2020). LD decayed rapidly, to $r^{2}<0.10$ by approximately $12 \mathrm{~kb}$, almost identical to the rate reported in a population of $P$. infestans (AyalaUsma et al. 2020), and comparable to rates reported in three plantpathogenic fungi: Parastagonospora nodoroum, Zymoseptoria tritici, and Pyrenophora teres (Anke et al. 2020; Gao et al. 2016; Hartmann et al. 2017). Background levels of LD $\left(r^{2}=0.04\right)$, however, were only reached at approximately $400 \mathrm{~kb}$, indicating the need for a fairly large candidate gene search space in GWAS.

We expected mating type to be easy to map via GWAS, since it is known to have a simple genetic architecture, represented a binary phenotype with both classes evenly distributed in our population, and was unassociated with population structure (Fig. 4). Indeed, we found strong statistical support for a single mating type locus on scaffold 4 (Fig. 4; Supplementary Fig. S3), the same region
A

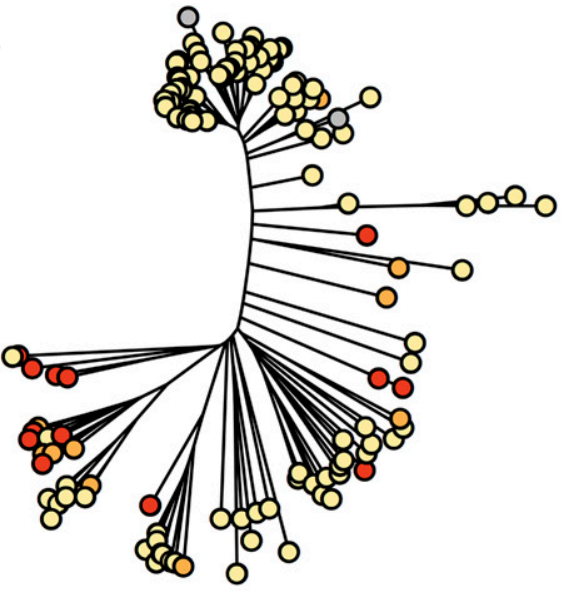

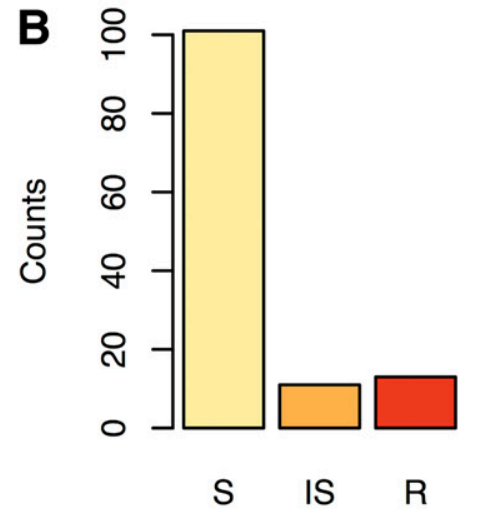

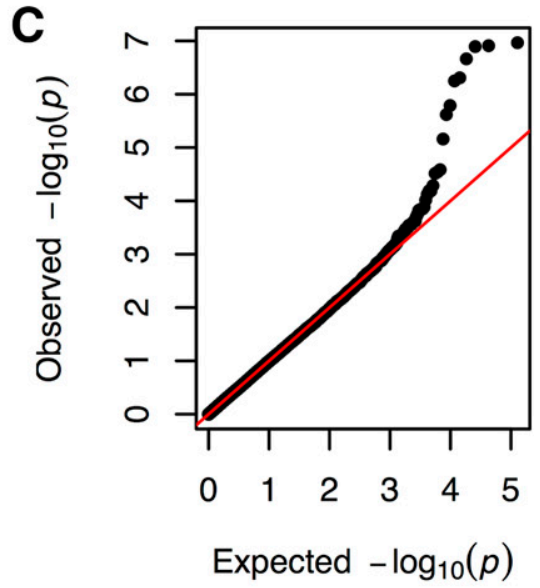

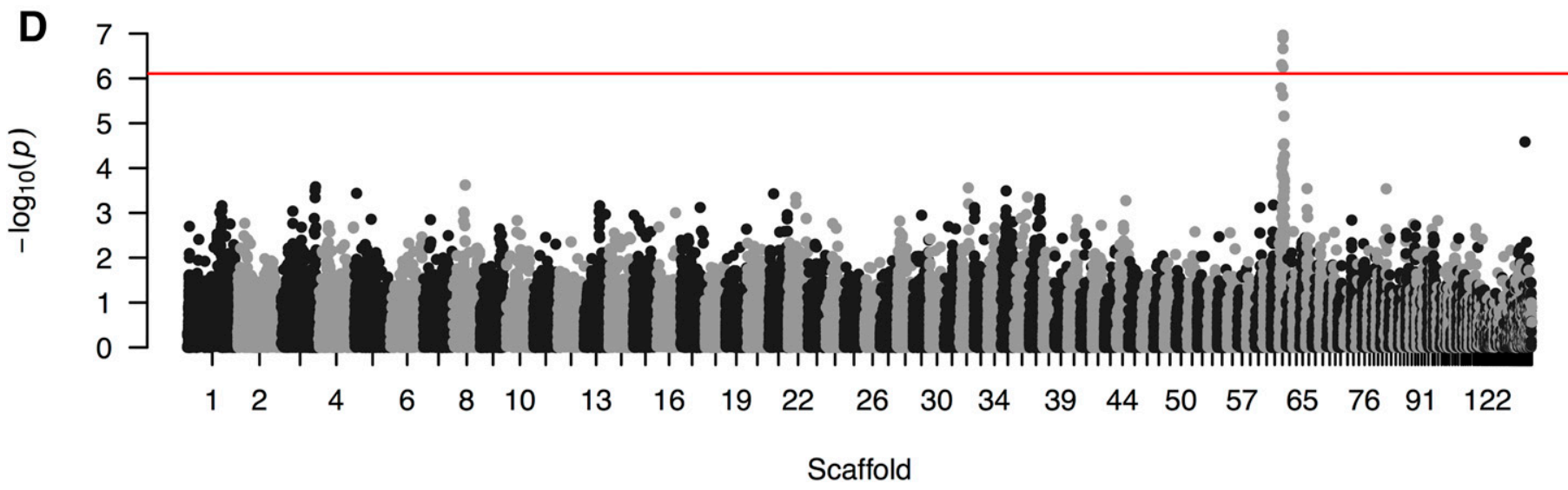

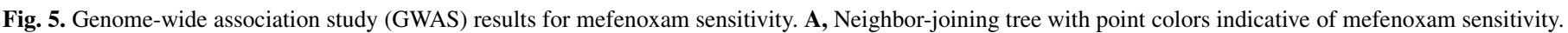

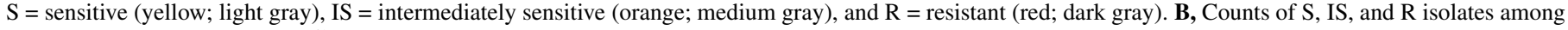

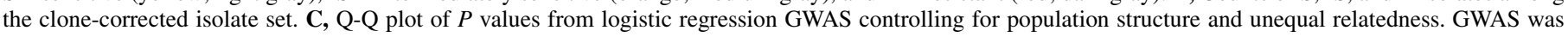

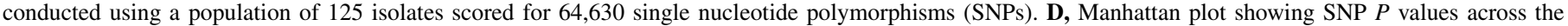
genome from logistic regression GWAS for mefenoxam sensitivity. 
identified in previous studies (Carlson et al. 2017; Lamour et al. 2012).

Previous evidence from experimental crosses supports a genetic model where mating type inheritance behaves as in an XY sex determination system, where, in the case of $P$. capsici, A2 isolates are the heterozygous (i.e., XY) type and A1 isolates are the homozygous type (i.e., XX) (Carlson et al. 2017; Fabritius and Judelson 1997). Our results are consistent with this hypothesis, as A1 isolates were predominantly homozygous and A2 isolates predominantly heterozygous at the 70 SNPs significantly associated with mating type. Other researchers have observed the $P$. capsici A2 mating type to be unstable (Hu et al. 2013, 2020), with evidence suggesting that mitotic loss of heterozygosity in the mating type region causes A2 isolates to switch mating type (Lamour et al. 2012). While we did not identify any A2 isolates that became self-fertile, a phenomenon that was recently reported $(\mathrm{Hu}$ et al. 2020), we did observe $13 \mathrm{~A} 2$ isolates that converted to A1 at an undetermined point over a period of several years. In addition, we identified mating type discrepancies within a single clonal lineage, consisting of one A1 and two A2 isolates. While the three isolates were genetically identical across most of the genome, the A1 isolate featured a high rate of discordant genotype calls compared with the
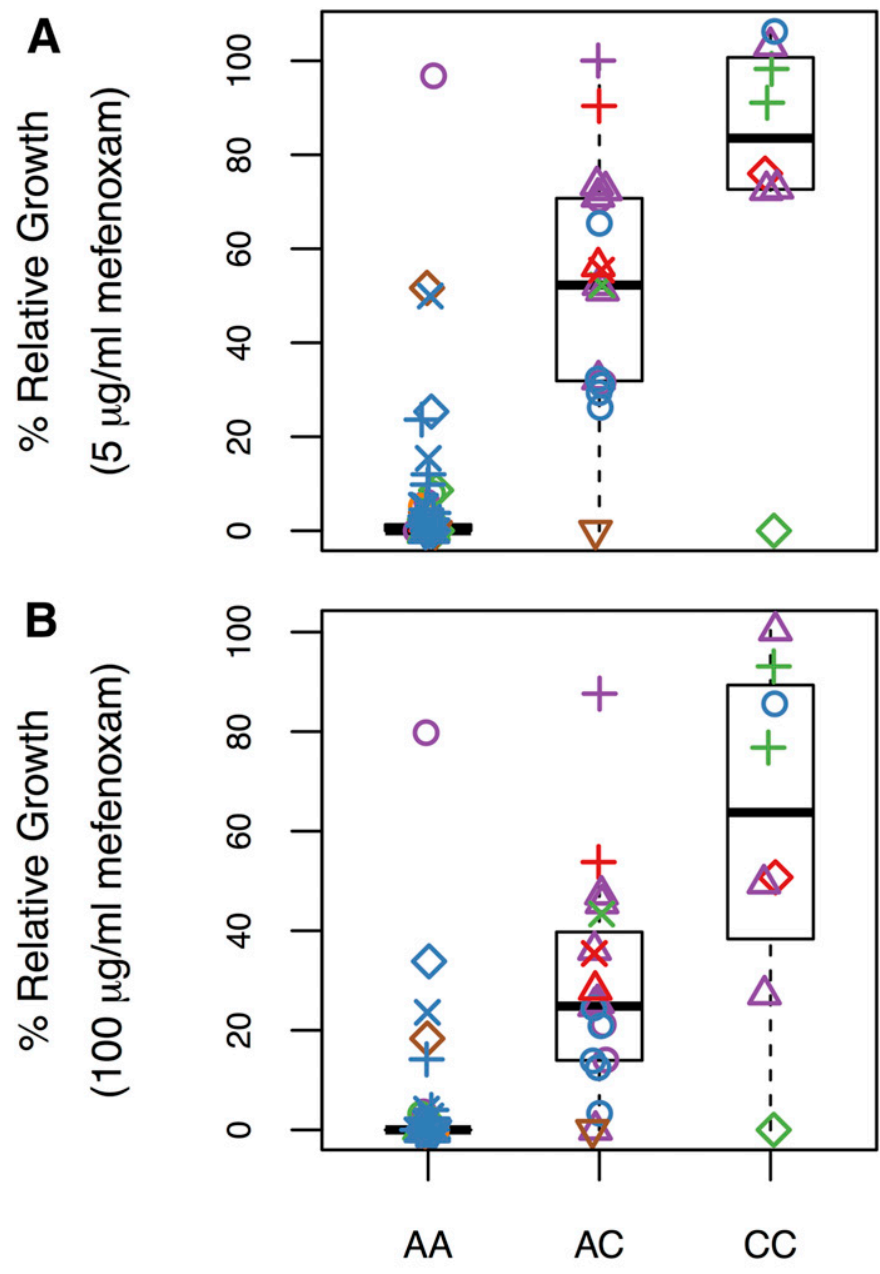

\section{Genotype}

Fig. 6. Boxplot showing the effect of the genotype at the peak single nucleotide polymorphism for mefenoxam insensitivity (S62_186715) on relative growth (RG) on amended media. Point colors and shapes are as in Figure 2. A, Effect of S62_186715 genotype on RG on mefenoxam at $5 \mu \mathrm{g} / \mathrm{ml}$. B, Effect of S62_186715 genotype on RG on mefenoxam at $100 \mu \mathrm{g} / \mathrm{ml}$.
A2 isolates at the mating type locus and linked genomic regions (Supplementary Fig. S4), consistent with a loss of heterozygosity event in this isolate resulting in the conversion of the A2determining haplotype. Because read depths were not halved in the mating type region compared with the rest of the genome, we hypothesize that this loss of heterozygosity occurred in a copyneutral manner such as gene conversion, as opposed to via a deletion event resulting in hemizygosity. Given what appears to be the high frequency of unidirectional switching from A2 to A1, it may be possible in a future experiment to fine-map the mating type locus by tracking loss of heterozygosity breakpoints in a collection of A2 isolates that have switched mating type.

We found the identification of mating type candidate genes to be challenging, due to the large number of genes annotated within $400 \mathrm{~kb}$ of the peak SNP and our lack of knowledge of the molecular mechanism behind mating type determination. Furthermore, in searching for reciprocal best hits between $P$. capsici and Plasmopara viticola, we were unable to identify any likely orthologs between the reported mating type regions of the two species. Presumably, the causal mating type determining gene or genes could encode any of a wide variety of proteins, such as enzymes in the metabolic pathways for $\alpha_{1}$ or $\alpha_{2}$, receptors involved in the recognition of $\alpha$ hormones, or transcription factors responsible for regulating mating type-specific expression levels. It is also possible that the causal gene or genes are not annotated in the $P$. capsici reference genome, and they may only be present on one of the two A2 haplotypes. Indeed, in P. infestans, the mating type locus was shown to contain a heteromorphic region where certain cDNA clones only hybridized to one of the two mating typespecific contigs (Randall et al. 2003).

Unlike mating type, mefenoxam sensitivity had never been mapped in $P$. capsici prior to this study. It also represented a more challenging phenotype to map, as it was confounded with population structure and exhibited an imbalance in the number of resistant versus sensitive isolates (Fig. 5). Nevertheless, by fitting a generalized mixed model correcting for population structure and unequal relatedness, we detected significantly associated SNPs in a region on scaffold 62 (Fig. 5; Supplementary Fig. S3). The allele associated with insensitivity at the peak SNP demonstrated an approximately additive effect (Fig. 6), consistent with segregation patterns in lab crosses that support an incompletely dominant gene conferring metalaxyl or mefenoxam insensitivity in $P$. capsici, P. infestans, and P. sojae (Bhat et al. 1993; Gisi and Cohen 1996; Lamour and Hausbeck 2000; Shattock 1988). It appears likely, however, that additional loci may play a role in decreased sensitivity in some isolates, as several individuals in our collection of isolates were homozygous for the sensitive allele at the peak SNP on scaffold 62 yet showed intermediate or high mefenoxam insensitivity. Similarly, in P. infestans, there is evidence of multiple loci involved in insensitivity to metalaxyl (Fabritius et al. 1997; Judelson and Roberts 1999).

Researchers have long assumed that the target site of phenylamide fungicides is RNA polymerase I (Griffith et al. 1992), since the mode of action of these chemicals in oomycetes involves inhibition of rRNA synthesis (Davidse et al. 1983; Wollgiehn et al. 1984). In P. infestans, however, attempts to associate mutations in subunits of RNA polymerase I with metalaxyl or mefenoxam insensitivity have been inconclusive (Matson et al. 2015; Randall et al. 2014). In our case, we did not identify any genes in the region of the GWAS signal on scaffold 62 encoding RNA polymerase I subunits. We did, however, identify several other plausible candidate genes (Supplementary Table S3). One gene, located within $15 \mathrm{~kb}$ of the peak SNP, is a homolog of yeast protein Rrp5, which is required for processing of pre-rRNA into the cleaved molecules that form the ribosome (Venema and Tollervey 1996). If mefenoxam does interfere with the function of Rrp5, the buildup of precursor rRNA molecules in the nucleolus could cause a depletion of total rRNA as these intermediate molecules are degraded by 
exonucleases or as feedback mechanisms result in decreased rRNA transcription. Alternatively, the causal gene at this locus could be one of the transporters located nearby, or perhaps even RNA polymerase III subunit Rpc4, also located within $15 \mathrm{~kb}$ of the peak SNP. Further investigation is required to determine which of these genes, if any, feature mutations that confer mefenoxam insensitivity in the isolates we assayed.

Conclusions. We genotyped a collection of NY P. capsici isolates at over 100,000 SNP markers and assayed them for their mating type and mefenoxam sensitivity. Results of population structure analyses were consistent with previous reports, showing limited gene flow between different fields and highlighting the importance of sexual reproduction in allowing inoculum to overwinter. Thirteen percent of the isolates we genotyped showed some degree of chromosomal copy number variation within their genomes, with linkage groups 6 and 17 featuring a particularly high rate of aneuploidy. GWAS confirmed previous results mapping the mating type locus to a region on scaffold 4 , and identified a novel locus associated with mefenoxam sensitivity on scaffold 62. These results provide a foundation for functional validation of candidate genes as well as molecular marker development for prediction of mating type and mefenoxam insensitivity. Furthermore, the panel of isolates we assembled and the dense marker data we generated represent a genetic resource that can be used for mapping other important traits in $P$. capsici, such as sensitivity to additional chemicals or virulence on economically important host plants.

\section{ACKNOWLEDGMENTS}

We thank Ali Cala, Santiago Tíjaro Bulla, Kimberly D'Arcangelo, Holly Lange, Charlotte Mineo, and Carolina Puentes Silva, all of whom provided valuable technical assistance in this project. We also thank Maryn Carlson, who collected a portion of the data and provided feedback and edits on an earlier version of this manuscript, as well as Di Wu and Dan Ilut, who offered bioinformatic assistance. In addition, we are grateful to Traven Bentley, Kishor Bhattarai, Elizabeth Buck, Mary Hausbeck, Brian Hill, Shaker Kousik, Margaret McGrath, Abby Seaman, and Darcy Telenko for providing cultures of isolates characterized in this study.

\section{LITERATURE CITED}

Anke, M., Moolhuijzen, P., Tao, Y., Mcilroy, J., Ellwood, S., Fowler, R. A., Platz, G. J., Kilian, A., and Snyman, L. 2020. Genomic regions associated with virulence in Pyrenophora teres f. teres identified by genome-wide association analysis and bi-parental mapping. Phytopathology 110:881-891.

Ashby, S. F. 1929. Strains and taxonomy of Phytophthora palmivora Butler (P. faberi Maubl.). Trans. Br. Mycol. Soc. 14:18-38.

Ayala-Usma, D., Danies, G., Myers, K. L., Bond, M. O., Romero-Navarro, J. A., Judelson, H. S., Restrepo, S., and Fry, W. 2020. Genome-wide association study identifies SNP markers associated with mycelial growth at 15,20 and $25^{\circ} \mathrm{C}$, mefenoxam resistance and mating type in Phytophthora infestans. Phytopathology 110:822-833.

Babadoost, M., and Pavon, C. 2013. Survival of oospores of Phytophthora capsici in soil. Plant Dis. 97:1478-1483.

Barchenger, D. W., Lamour, K. H., Sheu, Z.-M., Shrestha, S., Kumar, S., Lin, S.-W., Burlakoti, R., and Bosland, P. W. 2017. Intra- and intergenomic variation of ploidy and clonality characterize Phytophthora capsici on Capsicum sp. in Taiwan. Mycol. Prog. 16:955-963.

Bates, D., Mächler, M., Bolker, B., and Walker, S. 2015. Fitting linear mixedeffects models using lme4. J. Stat. Softw. 67:1-48.

Bhat, R. G., McBlain, B. A., and Schmitthenner, A. F. 1993. The inheritance of resistance to metalaxyl and to fluorophenylalanine in matings of homothallic Phytophthora sojae. Mycol. Res. 97:865-870.

Bowers, J. H., Papvizas, G. C., and Johnston, S. A. 1990. Effect of soil temperature and soil-water matric potential on the survival of Phytophthora capsici in natural soil. Plant Dis. 74:771.

Camacho, C., Coulouris, G., Avagyan, V., Ma, N., Papadopoulos, J., Bealer, K., and Madden, T. L. 2009. BLAST+: Architecture and applications. BMC Bioinformatics 10:421.

Carlson, M. O., Gazave, E., Gore, M. A., and Smart, C. D. 2017. Temporal genetic dynamics of an experimental, biparental field population of Phytophthora capsici. Front. Genet. 8:26.
Daggett, S. S., Knighton, J. E., and Therrien, C. D. 1995. Polyploidy among isolates of Phytophthora infestans from eastern Germany. J. Phytopathol. 143:419-422.

Danecek, P., Auton, A., Abecasis, G., Albers, C. A., Banks, E., DePristo, M. A., Handsaker, R. E., Lunter, G., Marth, G. T., Sherry, S. T., McVean, G., and Durbin, R. 2011. The variant call format and VCFtools. Bioinformatics 27:2156-2158.

Davidse, L. C., Hofman, A. E., and Velthuis, G. C. M. 1983. Specific interference of metalaxyl with endogenous RNA polymerase activity in isolated nuclei from Phytophthora megasperma f. sp. medicaginis. Exp. Mycol. 7:344-361

Dunn, A. R., Bruening, S. R., Grünwald, N. J., and Smart, C. D. 2014. Evolution of an experimental population of Phytophthora capsici in the field. Phytopathology 104:1107-1117.

Dunn, A. R., Milgroom, M. G., Meitz, J. C., McLeod, A., Fry, W. E., McGrath, M. T., Dillard, H. R., and Smart, C. D. 2010. Population structure and resistance to mefenoxam of Phytophthora capsici in New York State. Plant Dis. 94:1461-1468.

Dussert, Y., Legrand, L., Mazet, I. D., Couture, C., Piron, M.-C., Serre, R.-F., Bouchez, O., Mestre, P., Toffolatti, S. L., Giraud, T., and Delmotte, F. 2020. Identification of the first oomycete mating-type locus sequence in the grapevine downy mildew pathogen, Plasmopara viticola. Curr. Biol. doi.org/10.1016/j.cub.2020.07.057

Dussert, Y., Mazet, I. D., Couture, C., Gouzy, J., Piron, M.-C., Kuchly, C., Bouchez, O., Rispe, C., Mestre, P., and Delmotte, F. 2019. A high-quality grapevine downy mildew genome assembly reveals rapidly evolving and lineage-specific putative host adaptation genes. Genome Biol. Evol. 11:954-969.

Elshire, R. J., Glaubitz, J. C., Sun, Q., Poland, J. A., Kawamoto, K., Buckler, E. S., and Mitchell, S. E. 2011. A robust, simple genotyping-by-sequencing (GBS) approach for high diversity species. PLoS One 6:e19379.

Endelman, J. B. 2011. Ridge regression and other kernels for genomic selection with R package rrBLUP. Plant Genome 4:250-255.

Erwin, D. C., and Ribeiro, O. K. 1998. Phytophthora Diseases Worldwide. American Phytopathological Society, St. Paul, MN.

Fabritius, A.-L., and Judelson, H. S. 1997. Mating-type loci segregate aberrantly in Phytophthora infestans but normally in Phytophthora parasitica: Implications for models of mating-type determination. Curr. Genet. 32: 60-65.

Fabritius, A.-L., Shattock, R. C., and Judelson, H. S. 1997. Genetic analysis of metalaxyl insensitivity loci in Phytophthora infestans using linked DNA markers. Phytopathology 87:1034-1040.

Farrer, R. A., Henk, D. A., Garner, T. W. J., Balloux, F., Woodhams, D. C., and Fisher, M. C. 2013. Chromosomal copy number variation, selection and uneven rates of recombination reveal cryptic genome diversity linked to pathogenicity. PLoS Genet. 9:e1003703.

Fletcher, K., Gil, J., Bertier, L. D., Kenefick, A., Wood, K. J., Zhang, L., Reyes-Chin-Wo, S., Cavanaugh, K., Tsuchida, C., Wong, J., and Michelmore, R. 2019. Genomic signatures of heterokaryosis in the oomycete pathogen Bremia lactucae. Nat. Commun. 10:2645.

Foster, J. M., and Hausbeck, M. K. 2009. Resistance of pepper to Phytophthora crown, root, and fruit rot is affected by isolate virulence. Plant Dis. 94: 24-30.

Gao, Y., Liu, Z., Faris, J. D., Richards, J., Brueggeman, R. S., Li, X., Oliver, R. P., McDonald, B. A., and Friesen, T. L. 2016. Validation of genome-wide association studies as a tool to identify virulence factors in Parastagonospora nodorum. Phytopathology 106:1177-1185.

Gisi, U., and Cohen, Y. 1996. Resistance to phenylamide fungicides: A case study with Phytophthora infestans involving mating type and race structure. Annu. Rev. Phytopathol. 34:549-572.

Gisi, U., and Sierotzki, H. 2008. Fungicide modes of action and resistance in downy mildews. Eur. J. Plant Pathol. 122:157-167.

Glaubitz, J. C., Casstevens, T. M., Lu, F., Harriman, J., Elshire, R. J., Sun, Q., and Buckler, E. S. 2014. TASSEL-GBS: A high capacity genotyping by sequencing analysis pipeline. PLoS One 9:e90346.

Gogarten, S. M., Sofer, T., Chen, H., Yu, C., Brody, J. A., Thornton, T. A., Rice, K. M., and Conomos, M. P. 2019. Genetic association testing using the GENESIS R/Bioconductor package. Bioinformatics 35:5346-5348.

Granke, L. L., Quesada-Ocampo, L., Lamour, K., and Hausbeck, M. K. 2012. Advances in research on Phytophthora capsici on vegetable crops in the United States. Plant Dis. 96:1588-1600.

Granke, L. L., Windstam, S. T., Hoch, H. C., Smart, C. D., and Hausbeck, M. K. 2009. Dispersal and movement mechanisms of Phytophthora capsici sporangia. Phytopathology 99:1258-1264.

Griffith, J. M., Davis, A. J., and Grant, B. R. 1992. Target sites of fungicides to control oomycetes. Pages 69-100 in: Target Sites of Fungicide Action. W. Koller, ed. CRC Press, Boca Raton, FL.

Hartmann, F. E., Sánchez-Vallet, A., McDonald, B. A., and Croll, D. 2017. A fungal wheat pathogen evolved host specialization by extensive chromosomal rearrangements. ISME J. 11:1189-1204. 
Hausbeck, M. K., and Lamour, K. H. 2004. Phytophthora capsici on vegetable crops: Research progress and management challenges. Plant Dis. 88: 1292-1303.

Hill, W. G., and Robertson, A. 1968. Linkage disequilibrium in finite populations. Theor. Appl. Genet. 38:226-231.

Hu, J., Diao, Y., Zhou, Y., Lin, D., Bi, Y., Pang, Z., Fryxell, R. T., Liu, X., and Lamour, K. 2013. Loss of heterozygosity drives clonal diversity of Phytophthora capsici in China. PLoS One 8:e82691.

Hu, J., Shrestha, S., Zhou, Y., Mudge, J., Liu, X., and Lamour, K. 2020. Dynamic extreme aneuploidy (DEA) in the vegetable pathogen Phytophthora capsici and the potential for rapid asexual evolution. PLoS One 15: $\mathrm{e} 0227250$.

Hurtado-Gonzáles, O., Aragon-Caballero, L., Apaza-Tapia, W., Donahoo, R., and Lamour, K. 2008. Survival and spread of Phytophthora capsici in coastal Peru. Phytopathology 98:688-694.

Huson, D. H. 1998. SplitsTree: Analyzing and visualizing evolutionary data. Bioinformatics 14:68-73.

Jeffers, S. N., and Martin, S. B. 1986. Comparison of two media selective for Phytophthora and Pythium species. Plant Dis. 70:1038.

Jones, L. A., Worobo, R. W., and Smart, C. D. 2014. Plant-pathogenic oomycetes, Escherichia coli strains, and Salmonella spp. frequently found in surface water used for irrigation of fruit and vegetable crops in New York State. Appl. Environ. Microbiol. 80:4814-4820.

Judelson, H. S. 1997. Expression and inheritance of sexual preference and selfing potential in Phytophthora infestans. Fungal Genet. Biol. 21: 188-197.

Judelson, H. S., and Roberts, S. 1999. Multiple loci determining insensitivity to phenylamide fungicides in Phytophthora infestans. Phytopathology 89: 754-760

Kasuga, T., Bui, M., Bernhardt, E., Swiecki, T., Aram, K., Cano, L. M., Webber, J., Brasier, C., Press, C., Grünwald, N. J., Rizzo, D. M., and Garbelotto, M. 2016. Host-induced aneuploidy and phenotypic diversification in the sudden oak death pathogen Phytophthora ramorum. BMC Genomics 17:385.

Keinath, A. P. 2007. Sensitivity of populations of Phytophthora capsici from South Carolina to mefenoxam, dimethomorph, zoxamide, and cymoxanil. Plant Dis. 91:743-748.

Ko, W. H. 1978. Heterothallic Phytophthora: Evidence for hormonal regulation of sexual reproduction. Microbiology 107:15-18.

Kousik, C. S., and Keinath, A. P. 2008. First report of insensitivity to cyazofamid among isolates of Phytophthora capsici from the southeastern United States. Plant Dis. 92:979.

Lamour, K. H., and Hausbeck, M. K. 2000. Mefenoxam insensitivity and the sexual stage of Phytophthora capsici in Michigan cucurbit fields. Phytopathology 90:396-400.

Lamour, K. H., and Hausbeck, M. K. 2001. Investigating the spatiotemporal genetic structure of Phytophthora capsici in Michigan. Phytopathology 91: 973-980

Lamour, K. H., and Hausbeck, M. K. 2003. Effect of crop rotation on the survival of Phytophthora capsici in Michigan. Plant Dis. 87:841-845.

Lamour, K. H., Mudge, J., Gobena, D., Hurtado-Gonzales, O. P., Schmutz, J., Kuo, A., Miller, N. A., Rice, B. J., Raffaele, S., Cano, L. M., Bharti, A. K., Donahoo, R. S., Finley, S., Huitema, E., Hulvey, J., Platt, D., Salamov, A., Savidor, A., Sharma, R., Stam, R., Storey, D., Thines, M., Win, J., Haas, B. J., Dinwiddie, D. L., Jenkins, J., Knight, J. R., Affourtit, J. P., Han, C. S., Chertkov, O., Lindquist, E. A., Detter, C., Grigoriev, I. V., Kamoun, S., and Kingsmore, S. F. 2012. Genome sequencing and mapping reveal loss of heterozygosity as a mechanism for rapid adaptation in the vegetable pathogen Phytophthora capsici. Mol. Plant-Microbe Interact. 25:1350-1360.

Li, H., and Durbin, R. 2009. Fast and accurate short read alignment with Burrows-Wheeler transform. Bioinformatics 25:1754-1760.

Matson, M. E. H., Small, I. M., Fry, W. E., and Judelson, H. S. 2015. Metalaxyl resistance in Phytophthora infestans: Assessing role of RPA190 gene and diversity within clonal lineages. Phytopathology 105:1594-1600.

Ojika, M., Molli, S. D., Kanazawa, H., Yajima, A., Toda, K., Nukada, T., Mao, H., Murata, R., Asano, T., Qi, J., and Sakagami, Y. 2011. The second Phytophthora mating hormone defines interspecies biosynthetic crosstalk. Nat. Chem. Biol. 7:591-593.

Parada-Rojas, C. H., and Quesada-Ocampo, L. M. 2018. Analysis of microsatellites from transcriptome sequences of Phytophthora capsici and applications for population studies. Sci. Rep. 8:5194.

Paradis, E., Claude, J., and Strimmer, K. 2004. APE: Analyses of phylogenetics and evolution in R language. Bioinformatics 20:289-290.
Parra, G., and Ristaino, J. B. 2001. Resistance to mefenoxam and metalaxyl among field isolates of Phytophthora capsici causing Phytophthora blight of bell pepper. Plant Dis. 85:1069-1075.

Pembleton, L. W., Cogan, N. O. I., and Forster, J. W. 2013. StAMPP: An R package for calculation of genetic differentiation and structure of mixedploidy level populations. Mol. Ecol. Resour. 13:946-952.

Qi, J., Asano, T., Jinno, M., Matsui, K., Atsumi, K., Sakagami, Y., and Ojika, M. 2005. Characterization of a Phytophthora mating hormone. Science 309: 1828.

Quesada-Ocampo, L. M., Granke, L. L., Mercier, M. R., Olsen, J., and Hausbeck, M. K. 2011. Investigating the genetic structure of Phytophthora capsici populations. Phytopathology 101:1061-1073.

R Core Team. 2012. R: A Language and Environment for Statistical Computing. R Foundation for Statistical Computing, Vienna, Austria. http:// www.R-project.org/

Randall, E., Young, V., Sierotzki, H., Scalliet, G., Birch, P. R. J., Cooke, D. E. L., Csukai, M., and Whisson, S. C. 2014. Sequence diversity in the large subunit of RNA polymerase I contributes to mefenoxam insensitivity in Phytophthora infestans. Mol. Plant Pathol. 15:664-676.

Randall, T. A., Ah Fong, A., and Judelson, H. S. 2003. Chromosomal heteromorphism and an apparent translocation detected using a BAC contig spanning the mating type locus of Phytophthora infestans. Fungal Genet. Biol. 38:75-84.

Sakamoto, Y., Ishiguro, M., and Kitagawa, G. 1986. Akaike Information Criterion Statistics. D Reidel Publishing Company, Tokyo, Japan.

Sansome, E. 1961. Meiosis in the oogonium and antheridium of Pythium debaryanum Hesse. Nature 191:827-828.

Sansome, E., and Brasier, C. M. 1973. Diploidy and chromosomal structural hybridity in Phytophthora infestans. Nature 241:344-345.

Schlub, R. L. 1983. Epidemiology of Phytophthora capsici on bell pepper. J. Agric. Sci. 100:7-11.

Shattock, R. C. 1988. Studies on the inheritance of resistance to metalaxyl in Phytophthora infestans. Plant Pathol. 37:4-11.

Shrestha, S. K., Miyasaka, S. C., Shintaku, M., Kelly, H., and Lamour, K. 2017. Phytophthora colocasiae from Vietnam, China, Hawaii and Nepal: Intra- and inter-genomic variations in ploidy and a long-lived, diploid Hawaiian lineage. Mycol. Prog. 16:893-904.

Siegel, J. J., and Amon, A. 2012. New insights into the troubles of aneuploidy. Annu. Rev. Cell Dev. Biol. 28:189-214.

Silvar, C., Merino, F., and Díaz, J. 2006. Diversity of Phytophthora capsici in northwest Spain: Analysis of virulence, metalaxyl response, and molecular characterization. Plant Dis. 90:1135-1142.

Stacklies, W., Redestig, H., Scholz, M., Walther, D., and Selbig, J. 2007. pcaMethods - a bioconductor package providing PCA methods for incomplete data. Bioinformatics 23:1164-1167.

Sunshine, A. B., Payen, C., Ong, G. T., Liachko, I., Tan, K. M., and Dunham, M. J. 2015. The fitness consequences of aneuploidy are driven by conditiondependent gene effects. PLoS Biol. 13:e1002155.

Turner, S. D. 2014. qqman: An R package for visualizing GWAS results using Q-Q and Manhattan plots. bioRxiv 005165.

Venema, J., and Tollervey, D. 1996. RRP5 is required for formation of both $18 \mathrm{~S}$ and 5.8S rRNA in yeast. EMBO J. 15:5701-5714.

Wang, Z., Langston, D. B., Csinos, A. S., Gitaitis, R. D., Walcott, R. R., and Ji, P. 2009. Development of an improved isolation approach and simple sequence repeat markers to characterize Phytophthora capsici populations in irrigation ponds in southern Georgia. Appl. Environ. Microbiol. 75: 5467-5473.

Weir, B. S., and Cockerham, C. C. 1984. Estimating F-statistics for the analysis of population structure. Evolution 38:1358-1370.

Wollgiehn, R., Bräutigam, E., Schumann, B., and Erge, D. 1984. Effect of metalaxyl on the synthesis of RNA, DNA and protein in Phytophthora nicotianae. Z. Allg. Mikrobiol. 24:269-279.

Yoshida, K., Schuenemann, V. J., Cano, L. M., Pais, M., Mishra, B., Sharma, R., Lanz, C., Martin, F. N., Kamoun, S., Krause, J., Thines, M., Weigel, D., and Burbano, H. A. 2013. The rise and fall of the Phytophthora infestans lineage that triggered the Irish potato famine. eLife 2:e0731.

Zhang, C., Dong, S.-S., Xu, J.-Y., He, W.-M., and Yang, T.-L. 2019. PopLDdecay: A fast and effective tool for linkage disequilibrium decay analysis based on variant call format files. Bioinformatics 35:1786-1788.

Zhu, Y. O., Sherlock, G., and Petrov, D. A. 2016. Whole genome analysis of 132 clinical Saccharomyces cerevisiae strains reveals extensive ploidy variation. G3 (Bethesda) 6:2421-2434. 